\title{
QUASI-REPRESENTATIONS OF SURFACE GROUPS
}

\author{
JOSÉ R. CARRIÓN AND MARIUS DADARLAT
}

\begin{abstract}
By a quasi-representation of a group $G$ we mean an approximately multiplicative map of $G$ to the unitary group of a unital $C^{*}$-algebra. A quasirepresentation induces a partially defined map at the level $K$-theory.

In the early 90s Exel and Loring associated two invariants to almost-commuting pairs of unitary matrices $u$ and $v$ : one a $K$-theoretic invariant, which may be regarded as the image of the Bott element in $K_{0}\left(C\left(\mathbb{T}^{2}\right)\right)$ under a map induced by quasi-representation of $\mathbb{Z}^{2}$ in $U(n)$; the other is the winding number in $\mathbb{C} \backslash\{0\}$ of the closed path $t \mapsto \operatorname{det}(t v u+(1-t) u v)$. The so-called Exel-Loring formula states that these two invariants coincide if $\|u v-v u\|$ is sufficiently small.

A generalization of the Exel-Loring formula for quasi-representations of a surface group taking values in $U(n)$ was given by the second-named author. Here we further extend this formula for quasi-representations of a surface group taking values in the unitary group of a tracial unital $C^{*}$-algebra.
\end{abstract}

\section{INTRODUCTION}

Let $G$ be a discrete countable group. In [Dad12, Dad11] the second-named author studied the question of how deformations of the group $G$ (or of the group $C^{*}$-algebra $C^{*}(G)$ ) into the unitary group of a (unital) $C^{*}$-algebra $A$ act on the $K$-theory of the algebras $\ell^{1}(G)$ and $C^{*}(G)$. By a deformation we mean an almost-multiplicative map, a quasi-representation, which we will define precisely in a moment. Often, matrix-valued multiplicative maps are inadequate for detecting the $K$-theory of the aforementioned group algebras. In fact, if a countable, discrete, torsion free group $G$ satisfies the Baum-Connes conjecture, then a unital finite dimensional representation $\pi: C^{*}(G) \rightarrow M_{r}(\mathbb{C})$ induces the map $r \cdot \iota_{*}$ on $K_{0}\left(C^{*}(G)\right)$, where $\iota$ is the trivial representation of $G$ (see [Dad11, Proposition 3.2]). It turns out that almost-multiplicative maps detect $K$-theory quite well for large classes of groups: one can implement any group homomorphism of $K_{0}\left(C^{*}(G)\right)$ to $\mathbb{Z}$ on large swaths of $K_{0}\left(C^{*}(G)\right)$ using quasirepresentations (see [Dad11, Theorem 3.3]).

Knowing that quasi-representations may be used to detect $K$-theory, we turn to how it is that they act. An index theorem of Connes, Gromov and Moscovici in [CGM90] is very relevant to this topic, in the following context. Let $M$ be a closed

Date: June 15, 2013.

M.D. was partially supported by NSF grant \#DMS-1101305.

J.R.C. was partially supported by a Purdue Research Foundation grant. 
Riemannian manifold with fundamental group $G$ and let $D$ be an elliptic pseudodifferential operator on $M$. The equivariant index of $D$ is an element of $K_{0}\left(\ell^{1}(G)\right)$. Connes, Gromov and Moscovici showed that the push-forward of the equivariant index of $D$ under a quasi-representation of $G$ coming from parallel transport in an almost-flat bundle $E$ over $M$ is equal to the index of $D$ twisted by $E$.

At around the same time, Exel and Loring studied two invariants associated to pairs of almost-commuting scalar unitary matrices $u, v \in U(r)$. One is a $K$-theory invariant, which may be regarded as the push-forward of the Bott element $\beta$ in the $K_{0}$-group of $C\left(\mathbb{T}^{2}\right) \cong C^{*}\left(\mathbb{Z}^{2}\right)$ by a quasi-representation of $\mathbb{Z}^{2}$ into the unitary group $U(r)$. The Exel-Loring formula proved in [EL91] states that this invariant equals the winding number in $\mathbb{C} \backslash\{0\}$ of the path $t \mapsto \operatorname{det}((1-t) u v+t v u)$. An extension of this formula for almost commuting unitaries in a $C^{*}$-algebra of tracial rank one is due to $\mathrm{H}$. Lin and plays an important role in the classification theory of amenable $C^{*}$ algebras. In a different direction, the Exel-Loring formula was generalized in Dad12 to finite dimensional quasi-representations of a surface group using a variant of the index theorem of CGM90. Remark 2.7 discusses the Exel-Loring formula in more detail.

In Dad12], the second-named author used the Mishchenko-Fomenko index theorem to give a new proof and a generalization of the index theorem of Connes, Gromov and Moscovici that allows $C^{*}$-algebra coefficients. In this paper we use this generalization to address the question of how a quasi-representation $\pi$ of a surface group in the unitary group of a tracial $C^{*}$-algebra acts at the level of $K$-theory. We extend the Exel-Loring formula to a surface group $\Gamma_{g}$ (with canonical generators $\left.\alpha_{i}, \beta_{i}\right)$ and coefficients in a unital $C^{*}$-algebra $A$ with a trace $\tau$. Briefly, writing $K_{0}\left(\ell^{1}\left(\Gamma_{g}\right)\right) \cong \mathbb{Z}[1] \oplus \mathbb{Z} \mu\left[\Sigma_{g}\right]$ we have

$$
\tau\left(\pi_{\sharp}\left(\mu\left[\Sigma_{g}\right]\right)\right)=\frac{1}{2 \pi i} \tau\left(\log \left(\prod_{i=1}^{g}\left[\pi\left(\alpha_{i}\right), \pi\left(\beta_{i}\right)\right]\right)\right),
$$

where [1] is the $K_{0}$ class of the unit $1 \in \ell^{1}\left(\Gamma_{g}\right)$, [ $\left.\Sigma_{g}\right]$ is the fundamental class in $K$ homology of the genus $g$ surface $\Sigma_{g}$, and $\mu: K_{0}\left(\Sigma_{g}\right) \rightarrow K_{0}\left(\ell^{1}(G)\right)$ is the $\ell^{1}$-version of the assembly map of Lafforgue. For a complete statement see Theorem 2.6. In the proof we make use of Chern-Weil theory for connections on Hilbert $A$-module bundles as developed by Schick Sch05 and the de la Harpe-Skandalis determinant dlHS84 to calculate the first Chern class of an almost-flat Hilbert module $C^{*}$-bundle associated to a quasi-representation (Theorem 5.4).

The paper is organized as follows. In Section 2 we define quasi-representations and the invariants we are interested in, and state our main result, Theorem 2.6. The invariants make use of the Mishchenko line bundle, which we discuss in Section 3. The push-forward of this bundle by a quasi-representation is considered in Section 4 Section 5 contains our main technical result, Theorem 5.4, which computes one of our invariants in terms of the de la Harpe-Skandalis determinant dlHS84. To obtain the formula given in the Theorem 2.6, we must work with concrete triangulations of 
oriented surfaces; this is contained in Section 6. Assembling these results in Section 7 yields a proof of Theorem 2.6 .

\section{The MAIN RESUlT}

In this section we state our main result. It depends on a result in Dad12 that we revisit. Let us provide some notation and definitions first.

Let $G$ be a discrete countable group and $A$ a unital $C^{*}$-algebra.

Definition 2.1. Let $\varepsilon>0$ and let $\mathcal{F}$ be a finite subset of $G$. An $(\mathcal{F}, \varepsilon)$-representation of $G$ in $U(A)$ is a function $\pi: G \rightarrow U(A)$ such that for all $s, t \in \mathcal{F}$ we have

- $\pi(1)=1$,

- $\left\|\pi\left(s^{-1}\right)-\pi(s)^{*}\right\|<\varepsilon$, and

- $\|\pi(s t)-\pi(s) \pi(t)\|<\varepsilon$.

We refer to the third condition by saying that $\pi$ is $(\mathcal{F}, \varepsilon)$-multiplicative. Let us note that the second condition follows from the other two if we assume that $\mathcal{F}$ is symmetric, i.e. $\mathcal{F}=\mathcal{F}^{-1}$. A quasi-representation is an $(\mathcal{F}, \varepsilon)$-representation where $\mathcal{F}$ and $\varepsilon$ are not necessarily specified.

A quasi-representation $\pi: G \rightarrow U(A)$ induces a map (also denoted $\pi$ ) of the Banach algebra $\ell^{1}(G)$ to $A$ by $\sum \lambda_{s} s \mapsto \sum \lambda_{s} \pi(s)$. This map is a unital linear contraction. We also write $\pi$ for the extension of $\pi$ to matrix algebras over $\ell^{1}(G)$.

2.2. Pushing-forward via quasi-representations. A group homomorphism $\pi: G \rightarrow$ $U(A)$ induces a map $\pi_{*}: K_{0}\left(\ell^{1}(G)\right) \rightarrow K_{0}(A)$ (via its Banach algebra extension). We think of a quasi-representation $\pi$ as inducing a partially defined map $\pi_{\sharp}$ at the level of $K$-theory, in the following sense. If $e$ is an idempotent in some matrix algebra over $\ell^{1}(G)$ such that $\left\|\pi(e)-\pi(e)^{2}\right\|<1 / 4$, then the spectrum of $\pi(e)$ is disjoint from the line $\{\operatorname{Re} z=1 / 2\}$. Writing $\chi$ for the characteristic function of $\{\operatorname{Re} z>1 / 2\}$, it follows that $\chi(\pi(e))$ is an idempotent and we set

$$
\pi_{\sharp}(e)=[\chi(\pi(e))] \in K_{0}(A) .
$$

For an element $x$ in $K_{0}\left(\ell^{1}(G)\right)$ we make a choice of idempotents $e_{0}$ and $e_{1}$ in some matrix algebra over $\ell^{1}(G)$ such that $x=\left[e_{0}\right]-\left[e_{1}\right]$. If $\left\|\pi\left(e_{i}\right)-\pi\left(e_{i}\right)^{2}\right\|<1 / 4$ for $i \in\{0,1\}$, write $\pi_{\sharp}(x)=\pi_{\sharp}\left(e_{0}\right)-\pi_{\sharp}\left(e_{1}\right)$. The choice of idempotents is largely inconsequential: given two choices of representatives one finds that if $\pi$ is multiplicative enough, then both choices yield the same element of $K_{0}(A)$.

Of course, the more multiplicative $\pi$ is, the more elements of $K_{0}\left(\ell^{1}(G)\right)$ we can push-forward into $K_{0}(A)$.

2.3. An index theorem. Fix a closed oriented Riemannian surface $M$ and let $G$ be its fundamental group. Fix also a unital $C^{*}$-algebra $A$ with a tracial state $\tau$. Write $K_{0}(M)$ for $K K(C(M), \mathbb{C})$. Because the assembly map $\mu: K_{0}(M) \rightarrow K_{0}\left(\ell^{1}(G)\right)$ is known to be an isomorphism in this case Laf02, we have

$$
K_{0}\left(\ell^{1}(G)\right) \cong \mathbb{Z}[1] \oplus \mathbb{Z} \mu[M]
$$


where $[M]$ is the fundamental class of $M$ in $K_{0}(M)$ BMV05, Lemma 7.9] and [1] is the class of the unit of $\ell^{1}(G)$. Since we are interested in how a quasi-representation of $G$ acts on $K_{0}\left(\ell^{1}(G)\right)$, we would like to study push-forward of the generator $\mu[M]$ by a quasi-representation.

2.3.1. Consider the universal cover $\widetilde{M} \rightarrow M$ and the diagonal action of $G$ on $\widetilde{M} \times \ell^{1}(G)$ giving rise to the so-called Mishchenko line bundle $\ell, \widetilde{M} \times_{G} \ell^{1}(G) \rightarrow M$. We will discuss it in more detail in Section 3 , where we will give a description of it as the class of a specific idempotent $e$ in some matrix algebra over $C(M) \otimes \ell^{1}(G)$.

If $\pi$ is a quasi-representation of $G$ in $U(A)$, then $\operatorname{id}_{C(M)} \otimes \pi$ is an almost-multiplicative unital linear contraction on $C(M) \otimes \ell^{1}(G)$ with values in $C(M) \otimes A$. Assuming that $\pi$ is sufficiently multiplicative, we may define the push-forward of the idempotent $e$ by $\operatorname{id}_{C(M)} \otimes \pi$, just as in Section 2.2 . We set

$$
\ell_{\pi}:=\left(\operatorname{id}_{C(M)} \otimes \pi\right)_{\sharp}(\ell):=\left(\operatorname{id}_{C(M)} \otimes \pi\right)_{\sharp}(e) \in K_{0}(C(M) \otimes A) .
$$

Let $D$ be an elliptic operator on $M^{n}$ and let $\mu[D] \in K_{0}\left(\ell^{1}(G)\right)$ be its image under the assembly map. Let $q_{0}$ and $q_{1}$ be idempotents in some matrix algebra over $\ell^{1}(G)$ such that $\mu[D]=\left[q_{0}\right]-\left[q_{1}\right]$ and write $\pi_{\sharp}(\mu[D]):=\pi_{\sharp}\left(q_{0}\right)-\pi_{\sharp}\left(q_{1}\right)$. By $\operatorname{Dad} 12$, Corollary 3.8], if $\pi: G \rightarrow A$ is sufficiently multiplicative, then

$$
\tau\left(\pi_{\sharp}(\mu[D])\right)=(-1)^{n(n+1) / 2}\left\langle\left(p_{!} \operatorname{ch}(\sigma(D)) \cup \operatorname{Td}(T M \otimes \mathbb{C}) \cup \operatorname{ch}_{\tau}\left(\ell_{\pi}\right),[M]\right\rangle,\right.
$$

where $p: T M \rightarrow M$ is the canonical projection, $\operatorname{ch}(\sigma(D))$ is the Chern character of the symbol of $D, \operatorname{Td}\left(T_{\mathbb{C}} M\right)$ is the Todd class of the complexified tangent bundle, and $[M]$ is the fundamental homology class of $M$. Set $\alpha=(-1)^{n(n+1) / 2}\left(p_{!} \operatorname{ch}(\sigma(D)) \cup\right.$ $\operatorname{Td}(T M \otimes \mathbb{C})$. Then (1) becomes

$$
\tau\left(\pi_{\sharp}(\mu[D])\right)=\left\langle\alpha \cup \operatorname{ch}_{\tau}\left(\ell_{\pi}\right),[M]\right\rangle=\left\langle\operatorname{ch}_{\tau}\left(\ell_{\pi}\right), \alpha \cap[M]\right\rangle,
$$

On the other hand, it follows from the Atiyah-Singer index theorem that the Chern character in homology ch: $K_{0}(M) \rightarrow H_{*}(M ; \mathbb{Q})$ is given by

$$
\operatorname{ch}[D]=\left((-1)^{n(n+1) / 2} p_{!} \operatorname{ch}(\sigma(D)) \cup \operatorname{Td}\left(T_{\mathbb{C}} M\right)\right) \cap[M]=\alpha \cap[M] .
$$

It follows that

$$
\tau\left(\pi_{\sharp}(\mu[D])\right)=\left\langle\operatorname{ch}_{\tau}\left(\ell_{\pi}\right), \operatorname{ch}[D]\right\rangle
$$

In the case of surfaces this formula specializes to the following statement.

Theorem 2.4 (cf. Dad12, Corollary 3.8]). Let $M$ be a closed oriented Riemannian surface of genus $g$ with fundamental group $G$. Let $q_{0}$ and $q_{1}$ be idempotents in some matrix algebra over $\ell^{1}(G)$ such that $\mu[M]=\left[q_{0}\right]-\left[q_{1}\right]$. Then there exist a finite subset $\mathcal{G}$ of $G$ and $\omega>0$ satisfying the following.

Let $A$ be a unital $C^{*}$-algebra with a tracial state $\tau$ and let $\pi: G \rightarrow U(A)$ be a $(\mathcal{G}, \omega)$-representation. Write $\pi_{\sharp}(\mu[M]):=\pi_{\sharp}\left(q_{0}\right)-\pi_{\sharp}\left(q_{1}\right)$. Then

$$
\tau\left(\pi_{\sharp}(\mu[M])\right)=\left\langle\operatorname{ch}_{\tau}\left(\ell_{\pi}\right),[M]\right\rangle .
$$


Here $\operatorname{ch}_{\tau}: K_{0}(C(M) \otimes A) \rightarrow H^{2}(M, \mathbb{R})$ is a Chern character associated to $\tau$ (see Section 5 , and $[M] \in H_{2}(M, \mathbb{R})$ is the fundamental class of $M$.

Proof. Given another pair of idempotents $q_{0}^{\prime}, q_{1}^{\prime}$ in some matrix algebra over $\ell^{1}(G)$ such that $\mu[M]=\left[q_{0}^{\prime}\right]-\left[q_{1}^{\prime}\right]$, there is an $\omega_{0}>0$ such that if $0<\omega<\omega_{0}$, then for any $(\mathcal{G}, \omega)$-representation $\pi$ we have $\pi_{\sharp}\left(q_{0}\right)-\pi_{\sharp}\left(q_{1}\right)=\pi_{\sharp}\left(q_{0}^{\prime}\right)-\pi_{\sharp}\left(q_{1}^{\prime}\right)$. We are therefore free to prove the theorem for a convenient choice of idempotents.

It is known that the fundamental class of $M$ in $K_{0}(M)$ coincides with $\left[\bar{\partial}_{g}\right]+(g-$ 1) $[\iota]$ where $\bar{\partial}_{g}$ is the Dolbeault operator on $M$ and $\iota: C(M) \rightarrow \mathbb{C}$ is a character (see BMV05, Lemma 7.9]). Let $e_{0}, e_{1}, f_{0}, f_{1}$ be idempotents in some matrix algebra over $\ell^{1}(G)$ such that

$$
\mu\left[\bar{\partial}_{g}\right]=\left[e_{0}\right]-\left[e_{1}\right] \text { and } \mu[\iota]=\left[f_{0}\right]-\left[f_{1}\right] .
$$

(This gives an obvious choice of idempotents $q_{0}^{\prime}$ and $q_{1}^{\prime}$ in some matrix algebra over $\ell^{1}(G)$ so that $\mu[M]=\left[q_{0}^{\prime}\right]-\left[q_{1}^{\prime}\right]$.) We want to prove that

$$
\tau\left(\pi_{\sharp}(\mu(z))\right)=\left\langle\operatorname{ch}_{\tau}\left(\ell_{\pi}\right), \operatorname{ch}(z)\right\rangle
$$

for $z=[M] \in K_{0}(M)$. Because of the additivity of this last equation, the fact that $[M]=\left[\bar{\partial}_{g}\right]+(g-1)[\iota]$, and Eq. $(2)$, it is enough to prove that

$$
\tau\left(\pi_{\sharp}(\mu[\iota])\right)=\left\langle\operatorname{ch}_{\tau}\left(\ell_{\pi}\right), \operatorname{ch}[\iota]\right\rangle .
$$

By Dad12, Corollary 3.5]

$$
\tau\left(\pi_{\sharp}(\mu[\iota])\right)=\tau\left(\left\langle\ell_{\pi},[\iota] \otimes 1_{A}\right\rangle\right) .
$$

We can represent $\ell_{\pi}$ by a projection $f$ in matrices over $C(M, A)$. The definition of the Kasparov product implies that

$$
\left\langle\left[\ell_{\pi}\right],[\iota] \otimes 1_{A}\right\rangle=\iota_{*}[f]=\left[f\left(x_{0}\right)\right] \in K_{0}(A) .
$$

On the other hand, the definition of $\operatorname{ch}_{\tau}$ (see [Sch05, Definition 4.1]) implies that $\operatorname{ch}_{\tau}(f)=\tau\left(f\left(x_{0}\right)\right)+$ a term in $H^{2}(M, \mathbb{R})$. Since $\operatorname{ch}[\iota]=1 \in H_{0}(M, \mathbb{R})$, we get

$$
\left\langle\operatorname{ch}_{\tau}(f), \operatorname{ch}[\iota]\right\rangle=\tau\left(f\left(x_{0}\right)\right) .
$$

2.5. Statement of the main result. We will often write $\Sigma_{g}$ for the closed oriented surface of genus $g$ and $\Gamma_{g}$ for its fundamental group. It is well known that $\Gamma_{g}$ has a standard presentation

$$
\Gamma_{g}=\left\langle\alpha_{1}, \beta_{1}, \ldots, \alpha_{g}, \beta_{g} \mid \prod_{i=1}^{g}\left[\alpha_{i}, \beta_{i}\right]\right\rangle,
$$

where we write $[\alpha, \beta]$ for the multiplicative commutator $\alpha \beta \alpha^{-1} \beta^{-1}$.

Our main result is the following. 
Theorem 2.6. Let $g \geq 1$ be an integer and let $q_{0}$ and $q_{1}$ be idempotents in some matrix algebra over $\ell^{1}\left(\Gamma_{g}\right)$ such that $\mu\left[\Sigma_{g}\right]=\left[q_{0}\right]-\left[q_{1}\right] \in K_{0}\left(\ell^{1}\left(\Gamma_{g}\right)\right)$. There exists $\varepsilon_{0}>0$ and a finite subset $\mathcal{F}_{0}$ of $\Gamma_{g}$ such that for every $0<\varepsilon<\varepsilon_{0}$ and every finite subset $\mathcal{F} \supseteq \mathcal{F}_{0}$ of $\Gamma_{g}$ the following holds.

If $A$ is a unital $C^{*}$-algebra with a trace $\tau$ and $\pi: \Gamma_{g} \rightarrow U(A)$ is an $(\mathcal{F}, \varepsilon)$ representation, then

$$
\tau\left(\pi_{\sharp}\left(\mu\left[\Sigma_{g}\right]\right)\right)=\frac{1}{2 \pi i} \tau\left(\log \left(\prod_{i=1}^{g}\left[\pi\left(\alpha_{i}\right), \pi\left(\beta_{i}\right)\right]\right)\right),
$$

where $\pi_{\sharp}\left(\mu\left[\Sigma_{g}\right]\right):=\pi_{\sharp}\left(q_{0}\right)-\pi_{\sharp}\left(q_{1}\right)$.

The rest of the paper is devoted to the proof.

Remark 2.7. The case when $g=1$ and $A=M_{n}(\mathbb{C})$ recovers the Exel-Loring formula of [EL91]. As mentioned in the introduction, this formula states that two integer-valued invariants $\kappa(u, v)$ and $\omega(u, v)$ associated to a pair unitary matrices $u, v \in U(n)$ coincide as long as $\left\|[u, v]-1_{n}\right\|<c$, where $c$ is a small constant that is independent $n$. Exel observed (in [Exe93, Lemma 3.1]) that the "winding-number" invariant $\omega(u, v)$ equals

$$
\frac{1}{2 \pi i} \operatorname{tr}(\log ([u, v]))
$$

This corresponds to the right-hand side of (5).

The " $K$-theory invariant" $\kappa(u, v)$ is an element in the $K_{0}$-group of $M_{n}(\mathbb{C})$ and is regarded as an integer after identifying this group with $\mathbb{Z}$ (the isomorphism given by the usual trace on $M_{n}(\mathbb{C})$ ). This invariant was first introduced by Loring in [Lor88]. We briefly recall its definition.

Recall that $K_{0}\left(C\left(\mathbb{T}^{2}\right)\right) \cong \mathbb{Z}[1] \oplus \mathbb{Z}[\beta]$ where [1] is the class of the unit of $C\left(\mathbb{T}^{2}\right)$ and $\beta$ is the Bott element. Given unitaries $U$ and $V$ in a unital $C^{*}$-algebra $B$, one defines a self-adjoint matrix

$$
e(U, V)=\left(\begin{array}{cc}
f(V) & g(V)+h(V) U^{*} \\
g(V)+U h(V) & 1-f(V)
\end{array}\right)
$$

where $f, g$, and $h$ are certain continuous functions on the circle. These are chosen in such a way that when $U=e^{2 \pi i x}, V=e^{2 \pi i y} \in C\left(\mathbb{T}^{2}\right)$ we have that $e(U, V)$ is idempotent and has $K_{0}$-class [1] $+\beta$ (cf. [Rie81, Lor88]).

We assume that $\left\|[u, v]-1_{n}\right\|$ is small enough so that the corresponding matrix $e(u, v)$ is nearly idempotent; in particular its spectrum does not contain $1 / 2$. Writing $\chi$ for the characteristic function of $\{\operatorname{Re} z>1 / 2\}$, we have that $\chi(e(u, v))$ is a projection in $M_{n}(\mathbb{C})$. Define

$$
\kappa(u, v)=\operatorname{tr}(\chi(e(u, v)))-n .
$$

Subtracting $n$ means cancelling-out the $K$-theoretic contribution of [1], leaving only the contribution of the push-forward of $\beta$ under a quasi-representation determined 
by $u$ and $v$. (Proposition 2.8 makes this statement precise.) This corresponds to the left-hand side of (5).

The formula (5) also recovers its extension by H. Lin Lin11 for $C^{*}$-algebras of tracial rank one. Lin's strategy was a reduction to the finite-dimensional case of [EL91] using approximation techniques.

The following proposition says that we may associate quasi-representations with unitaries that nearly satisfy the group relation in the standard presentation of $\Gamma_{g}$ mentioned above. The proof is in Section 7 .

Proposition 2.8. For every $\varepsilon>0$ and every finite subset $\mathcal{F}$ of $\Gamma_{g}$ there is a $\delta>0$ such that if $A$ is a unital $C^{*}$-algebra with a trace $\tau$ and $u_{1}, v_{1}, \ldots, u_{g}, v_{g}$ are unitaries in A satisfying

$$
\left\|\prod_{i=1}^{g}\left[u_{i}, v_{i}\right]-1\right\|<\delta,
$$

then there exists an $(\mathcal{F}, \varepsilon)$-representation $\pi: \Gamma_{g} \rightarrow U(A)$ with $\pi\left(\alpha_{i}\right)=u_{i}$ and $\pi\left(\beta_{i}\right)=v_{i}$, for all $i \in\{1, \ldots, g\}$.

Example. To revisit a classic example, consider the noncommutative 2-torus $A_{\theta}$, regarded as the universal $C^{*}$-algebra generated by unitaries $u$ and $v$ with $[v, u]=$ $e^{2 \pi i \theta} \cdot 1$. This is a tracial unital $C^{*}$-algebra. If $\theta$ is small enough, we may apply Proposition 2.8 and Theorem 2.6 to obtain

$$
\tau\left(\pi_{\sharp}(\beta)\right)=\frac{1}{2 \pi i} \tau\left(\log e^{-2 \pi i \theta}\right)=-\theta
$$

where $\beta \in K_{0}\left(C\left(\mathbb{T}^{2}\right)\right)$ is the Bott element, $\tau$ is a unital trace of $A_{\theta}$, and $\pi: \mathbb{Z}^{2} \rightarrow$ $U\left(A_{\theta}\right)$ is a quasi-representation obtained from Proposition 2.8

\section{The Mishchenko Line Bundle}

Recall our setup: $M$ is a closed oriented surface with fundamental group $G$ and universal cover $p: \widetilde{M} \rightarrow M$. In this section we give a picture of the Mishchenko line bundle that will enable us to explicitly describe its push-forward by a quasirepresentation.

The Mishchenko line bundle is the bundle $\widetilde{M} \times{ }_{G} \ell^{1}(G) \rightarrow M$, obtained from $\widetilde{M} \times \ell^{1}(G)$ by passing to the quotient with respect to the diagonal action of $G$. We write $\ell$ for its class in $K_{0}\left(C(M) \otimes \ell^{1}(G)\right)$.

3.1. Triangulations and the edge-path group. We adapt a construction found in the appendix of [PS90]. It is convenient to work with a triangulation $\Lambda$ of $M$. Let $\Lambda^{(0)}=\left\{x_{0}, \ldots, x_{N-1}\right\}$ be the 0 -skeleton of $\Lambda$ and $\Lambda^{(1)}$ be the 1-skeleton. To each edge we assign an element of $G$ as follows. Fix a root vertex $x_{0}$ and a maximal (spanning) tree $T$ in $\Lambda$. Let $\gamma_{i}$ be the unique path along $T$ from $x_{0}$ to $x_{i}$, and for two adjacent vertices $x_{i}$ and $x_{j}$ let $x_{i} x_{j}$ be the (directed) edge from $x_{i}$ to $x_{j}$. For two such adjacent vertices, write $s_{i j} \in G$ for the class of the loop $\gamma_{i} * x_{i} x_{j} * \gamma_{j}^{-1}$. 




(A)

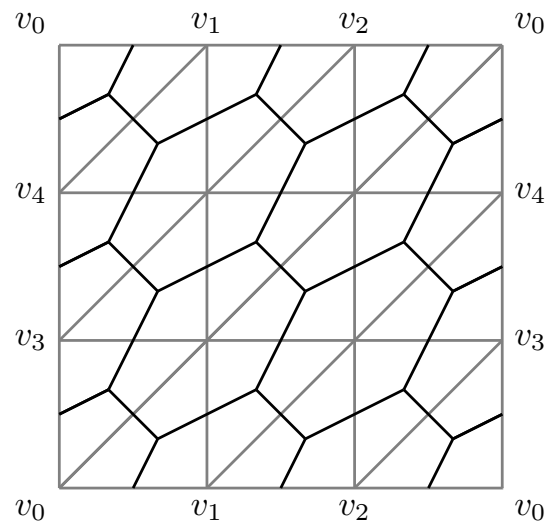

(B)

Figure 1. (A) Dual cell blocks in a simplex $\sigma=\left\langle v_{i_{0}}, v_{i_{1}}, v_{i_{2}}\right\rangle$. (B) A triangulation of $\mathbb{T}^{2}$ with the dual cell structure highlighted.

Let $\mathcal{F}$ be the (finite) set $\left\{s_{i j}\right\}$. For example, if $M=\mathbb{T}^{2}$ so that $G=\mathbb{Z}^{2}=$ $\langle\alpha, \beta:[\alpha, \beta]=1\rangle$, we have $\mathcal{F}=\left\{1, \alpha^{ \pm 1}, \beta^{ \pm 1},(\alpha \beta)^{ \pm 1}\right\}$ for the triangulation and tree pictured in Figure 3 (on page 20)

Definition 3.2. For a vertex $x_{i_{k}}$ in a 2 -simplex $\sigma=\left\langle x_{i_{0}}, x_{i_{1}}, x_{i_{2}}\right\rangle$ of $\Lambda$, define the dual cell block to $x_{i_{k}}$

$$
U_{i_{k}}^{\sigma}:=\left\{\sum_{l=0}^{2} t_{l} x_{i_{l}}: t_{l} \geq 0, \sum_{l=0}^{2} t_{l}=1, \text { and } t_{i_{k}} \geq t_{l} \text { for all } l\right\} .
$$

Define the dual cell to the vertex $x_{i} \in \Lambda^{(0)}$ by

$$
U_{i}=\cup\left\{U_{i}^{\sigma}: x_{i} \in \sigma\right\} .
$$

Let $U_{i j}^{\sigma}=U_{i}^{\sigma} \cap U_{j}^{\sigma}$ etc. (See Figure 1.)

Since $p: \widetilde{M} \rightarrow M$ is a covering space of $M$, we may fix an open cover of $M$ such that for every element $V$ of this cover, $p^{-1}(V)$ is a disjoint union of open subsets of $\widetilde{M}$, each of which is mapped homeomorphically onto $V$ by $p$. We require that $\Lambda$ be fine enough so that every dual cell $U_{i}$ is contained in some element of this cover.

Lemma 3.3. The Mishchenko line bundle $\widetilde{M} \times_{G} \ell^{1}(G) \rightarrow M$ is isomorphic to the bundle $E$ obtained from the disjoint union $\bigsqcup U_{i} \times \ell^{1}(G)$ by identifying $(x, a)$ with $\left(x, s_{i j} a\right)$ whenever $x \in U_{i} \cap U_{j}$.

Proof. Lift $x_{0}$ to a vertex $\widetilde{x}_{0}$ in $\widetilde{M}$. By the unique path-lifting property, every path $\gamma_{i}$ lifts (uniquely) to a path $\widetilde{\gamma}_{i}$ from $\widetilde{x}_{0}$ to a lift $\widetilde{x}_{i}$ of $x_{i}$. In this way lift $T$ to a 
tree $\widetilde{T}$ in $\widetilde{M}$. Each $U_{i}$ also lifts to a dual cell to $\widetilde{x}_{i}$, denoted $\widetilde{U}_{i}$, which $p$ maps homeomorphically onto $U_{i}$.

We first describe the cocycle (transition functions) for the Mishchenko line bundle. Identify the fundamental group $G$ of $M$ with the group of deck transformations of $\widetilde{M}$; see for example [Hat02, Proposition 1.39]. Use this to write $p^{-1}\left(U_{i}\right)$ as the disjoint union $\sqcup\left\{s \widetilde{U}_{i}: s \in G\right\}$. Consider the isomorphism $\Phi_{i}: p^{-1}\left(U_{i}\right) \times_{G} \ell^{1}(G) \rightarrow U_{i} \times \ell^{1}(G)$ described by the following diagram:

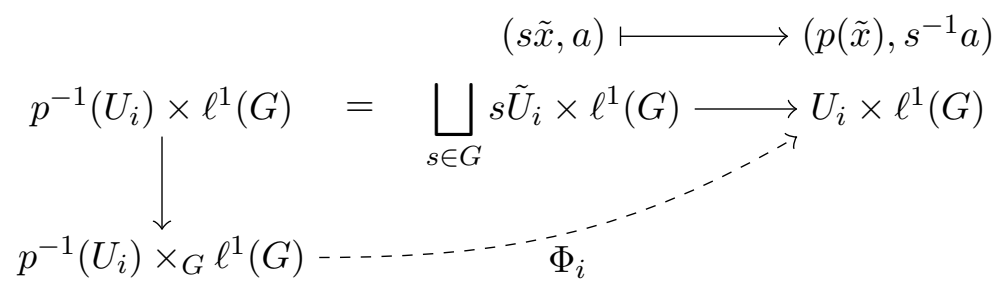

If $U_{i j}:=U_{i} \cap U_{j} \neq \emptyset$, we obtain the cocycle $\phi_{i j}: U_{i j} \rightarrow \operatorname{Aut}\left(\ell^{1}(G)\right)$ :

$$
\begin{gathered}
U_{i j} \times \ell^{1}(G) \stackrel{\Phi_{j}^{-1}}{\longrightarrow} p^{-1}\left(U_{i j}\right) \times_{G} \ell^{1}(G) \stackrel{\Phi_{i}}{\longrightarrow} U_{i j} \times \ell^{1}(G) \\
(x, a) \longmapsto\left(x, \phi_{i j}(x) a\right)
\end{gathered}
$$

Observe that $\widetilde{M} \times{ }_{G} \ell^{1}(G)$ is isomorphic to the bundle obtained from the disjoint union $\bigsqcup U_{i} \times \ell^{1}(G)$ by identifying $(x, a)$ with $\left(x, \phi_{i j}(x) a\right)$ whenever $x \in U_{i j}$. We only need to prove that $\phi_{i j}$ is constantly equal to $s_{i j}$.

Let $x \in U_{i j}$ and let $\widetilde{x} \in \widetilde{U}_{j}$ be a lift of $x$. Then $\Phi_{j}([\widetilde{x}, a])=(x, a)$. Because $p(\widetilde{x}) \in$ $U_{i j}$ there is a (unique) $s \in G$ such that $\widetilde{x} \in s \widetilde{U}_{i} \cap \widetilde{U}_{j} \neq \emptyset$. Thus $\Phi_{i}([\widetilde{x}, a])=\left(x, s^{-1} a\right)$. Now, the path $s \widetilde{\gamma}_{i} * s \widetilde{x}_{i} \widetilde{x}_{j} * \widetilde{\gamma}_{j}^{-1}$, starts at $s \widetilde{x}_{0}$ and ends at $\widetilde{x}_{0}$. Its projection in $M$ is the loop defining $s_{i j}$, so $s^{-1}=s_{i j}$. Thus $\phi_{i j}(x)=s_{i j}$.

3.4. The push-forward of the line bundle. We will need an open cover of $M$, so we dilate the dual cells $U_{i}$ to obtain one. Let $0<\delta<1 / 2$ and define $V_{i}^{\sigma}$ to be the $\delta$-neighborhood of $U_{i}^{\sigma}$ intersected with $\sigma$. As before, set $V_{i}=\bigcup_{\sigma} V_{i}^{\sigma}$. Let $\left\{\chi_{i}\right\}$ be a partition of unity subordinate to $\left\{V_{i}\right\}$.

By Lemma 3.3 the class of the Mishchenko line bundle in $K_{0}\left(C(M) \otimes \ell^{1}(G)\right)$, denoted earlier by $\ell$, corresponds to the class of the projection

$$
e:=\sum_{i, j} e_{i j} \otimes \chi_{i}^{1 / 2} \chi_{j}^{1 / 2} \otimes s_{i j} \in M_{N}(\mathbb{C}) \otimes C(M) \otimes \ell^{1}(G),
$$

where $\left\{e_{i j}\right\}$ are the canonical matrix units of $M_{N}(\mathbb{C})$ and $N$ is the number of vertices in $\Lambda$.

We may fix a pair of idempotents $q_{0}$ and $q_{1}$ in some matrix algebra over $\ell^{1}(G)$ satisfying $\left[q_{0}\right]-\left[q_{1}\right]=\mu[M] \in K_{0}\left(\ell^{1}(G)\right)$. Let $\omega>0$ be given by Theorem 2.4. (We may assume that $\omega<1 / 4$.) 
Fix $0<\varepsilon<\omega$ and an $(\mathcal{F}, \varepsilon)$-representation $\pi: G \rightarrow U(A)$. We recall the following notation from the introduction.

Notation 3.5. For an $(\mathcal{F}, \varepsilon)$-representation $\pi: G \rightarrow U(A)$ as above, let

$$
\ell_{\pi}:=\left(\operatorname{id}_{C(M)} \otimes \pi\right)_{\sharp}(e) .
$$

\section{Hilbert-module BUndLes AND QUASI-REPRESENTATIONS}

As mentioned in the introduction, in CGM90] a quasi-representation (with scalar values) of the fundamental group of a manifold is associated to an "almost-flat" bundle over the manifold. In this section we instead define a canonical bundle $E_{\pi}$ over $M$ associated with quasi-representation $\pi$. Its class in $K_{0}(C(M) \otimes A)$ will be the class $\ell_{\pi}$ of the push-forward of the Mishchenko line bundle by $\pi$. Our construction will be explicit enough so that we can use Chern-Weil theory for such bundles to analyze $\operatorname{ch}_{\tau}\left(\ell_{\pi}\right)$, see [Sch05].

Recall that $A$ is a $C^{*}$-algebra with trace $\tau$.

Definition. Let $X$ be a locally compact Hausdorff space. A Hilbert A-module bundle $W$ over $X$ is a topological space $W$ with a projection $W \rightarrow X$ such that the fiber over each point has the structure of a Hilbert $A$-module $V$, and with local trivializations $\left.W\right|_{U} \stackrel{\sim}{\longrightarrow} U \times V$ which are fiberwise Hilbert $A$-module isomorphisms.

We should point out that the $K_{0}$-group of the $C^{*}$-algebra $C(M) \otimes A$ is isomorphic to the Grothendieck group of isomorphism classes of finitely generated projective Hilbert $A$-module bundles over $M$. We identify the two groups.

4.1. Constructing bundles. We adapt a construction found in PS90].

First we define a family of maps $\left\{u_{i j}: U_{i j} \rightarrow \mathrm{GL}(A)\right\}$ satisfying

$$
\begin{aligned}
& u_{j i}(x)=u_{i j}^{-1}(x), \quad x \in U_{i j}, \\
& u_{i k}(x)=u_{i j}(x) u_{j k}(x), \quad x \in U_{i j k} .
\end{aligned}
$$

These maps will be then extended to a cocycle defined on the collection $\left\{V_{i j}\right\}$.

Following [PS90] we will find it convenient to fix a partial order $\mathbf{o}$ on the vertices of $\Lambda$ such that the vertices of each simplex form a totally ordered subset. We then call $\Lambda$ a locally ordered simplicial complex. One may always assume such an order exists by passing to the first barycentric subdivision of $\Lambda$ : if $\hat{\sigma}_{1}$ and $\hat{\sigma}_{2}$ are the barycenters of simplices $\sigma_{1}$ and $\sigma_{2}$ of $\Lambda$, define $\hat{\sigma}_{1}<\hat{\sigma}_{2}$ if $\sigma_{1}$ is a face of $\sigma_{2}$.

Consider a simplex $\sigma=\left\langle x_{i_{0}}, x_{i_{1}}, x_{i_{2}}\right\rangle$ (with vertices written in increasing o-order). Observe that in this case $U_{i_{0}}^{\sigma} \cap U_{i_{2}}^{\sigma}=U_{i_{0} i_{2}}^{\sigma}$ may be described using a single parameter $t_{1}$ :

$$
U_{i_{0} i_{2}}^{\sigma}=\left\{\sum_{l=0}^{2} t_{l} x_{i_{l}}: t_{0}=t_{2}=\frac{1-t_{1}}{2}: 0 \leq t_{1} \leq 1 / 3\right\} .
$$


Define

$$
\begin{aligned}
u_{i_{0} i_{1}}^{\sigma} & =\text { the constant function on } U_{i_{0} i_{1}}^{\sigma} \text { equal to } \pi\left(s_{i_{0} i_{1}}\right) \\
u_{i_{1} i_{2}}^{\sigma} & =\text { the constant function on } U_{i_{1} i_{2}}^{\sigma} \text { equal to } \pi\left(s_{i_{1} i_{2}}\right) \\
u_{i_{0} i_{2}}^{\sigma}\left(t_{1}\right) & =\left(1-3 t_{1}\right) \pi\left(s_{i_{0} i_{2}}\right)+3 t_{1} \pi\left(s_{i_{0} i_{1}}\right) \pi\left(s_{i_{1} i_{2}}\right), \quad 0 \leq t_{1} \leq 1 / 3 .
\end{aligned}
$$

Define $u_{i_{2} i_{0}}^{\sigma}$ etc. to be the pointwise inverse of $u_{i_{0} i_{2}}^{\sigma}$. For fixed $i$ and $j$, the maps $u_{i j}^{\sigma}: U_{i j}^{\sigma} \rightarrow \mathrm{GL}(A)$ define a map $u_{i j}: U_{i j} \rightarrow \mathrm{GL}(A)$. Indeed, if $x_{i} x_{j}$ is a common edge of two simplices $\sigma$ and $\sigma^{\prime}$, then $U_{i j}^{\sigma} \cap U_{i j}^{\sigma^{\prime}}$ is the barycenter of $\left\langle x_{i}, x_{j}\right\rangle$, where by definition both $u_{i j}^{\sigma}$ and $u_{i j}^{\sigma^{\prime}}$ take the value $\pi\left(s_{i j}\right)$. By construction the family $\left\{u_{i j}\right\}$ has the desired properties.

4.1.1. Recall the sets $V_{i}$ etc. from Section 3.4. To define the smooth transition function $v_{i_{0} i_{2}}^{\sigma}: V_{i_{0} i_{2}}^{\sigma} \rightarrow \mathrm{GL}(A)$ that will replace $u_{i_{0} i_{2}}^{\sigma}$, let us assume for simplicity that the simplex $\sigma$ is the triangle with vertices $v_{i_{0}}=(-1 / 2,0), v_{i_{1}}=(0,1)$, and $v_{i_{2}}=(1 / 2,0)$. (It may be helpful to consider Figure 1A.)

Define $v_{i_{0} i_{2}}^{\sigma}$ as follows:

$$
v_{i_{0} i_{2}}^{\sigma}(x, y)= \begin{cases}\pi\left(s_{i_{0} i_{1}}\right) \pi\left(s_{i_{1} i_{2}}\right), & 1 / 3-\delta \leq y \leq 1 / 3+\delta \\ \left(1-\frac{y}{1 / 3-\delta}\right) \pi\left(s_{i_{0} i_{2}}\right)+ & \\ +\frac{y}{1 / 3-\delta} \pi\left(s_{i_{0} i_{1}}\right) \pi\left(s_{i_{1} i_{2}}\right), & 0 \leq y \leq 1 / 3-\delta\end{cases}
$$

(so $v_{i_{0} i_{2}}^{\sigma}$ is constant along the horizontal segments in $V_{i_{0} i_{2}}$ ). The remaining two transition functions remain constant:

$$
\begin{aligned}
& v_{i_{0} i_{1}}^{\sigma}=\pi\left(s_{i_{0} i_{1}}\right) \\
& v_{i_{1} i_{2}}^{\sigma}=\pi\left(s_{i_{1} i_{2}}\right)
\end{aligned}
$$

Again, for fixed $i$ and $j$ the maps $v_{i j}^{\sigma}: V_{i j}^{\sigma} \rightarrow \mathrm{GL}(A)$ define a map $v_{i j}: V_{i j} \rightarrow \operatorname{GL}(A)$. Since $v_{i_{0} i_{2}}^{\sigma}$ is constant and equal to $\pi\left(s_{i_{0} i_{1}}\right) \pi\left(s_{i_{1} i_{2}}\right)$ in $V_{i_{0}} \cap V_{i_{2}} \cap V_{i_{2}}$, we indeed obtain a family $\left\{v_{i j}\right\}$ of transition functions.

Definition 4.2. The Hilbert $A$-module bundle $E_{\pi}$ is constructed from the disjoint union $\bigsqcup V_{i} \times A$ by identifying $(x, a)$ with $\left(x, v_{i j}(x) a\right)$ for $x$ in $V_{i j}$.

Proposition 4.3. The class of $E_{\pi}$ in $K_{0}(C(M) \otimes A)$ coincides with $\ell_{\pi}$, the class of the push-forward of e by $\operatorname{id}_{C(M)} \otimes \pi$ (see Section 3.4).

Proof. The bundle $E_{\pi}$ is a quotient of $\bigsqcup V_{i} \times A$ and from its definition it is clear that for each $i$ the quotient map is injective on $V_{i} \times A$. The restriction of the quotient map to $V_{i} \times A$ has an inverse, call it $\psi_{i}$, and $\psi_{i}$ is a trivialization of $\left.E_{\pi}\right|_{V_{i}}$. Recalling that $N$ is the number of vertices in $\Lambda$ (which is the same as the number of sets $V_{i}$ in the cover), we define an isometric embedding

$$
\begin{gathered}
\theta: E_{\pi} \rightarrow M \times A^{N} \\
{[x, a] \mapsto\left(\chi_{i}^{1 / 2}(x) \psi_{i}([x, a])\right)_{i=0}^{N-1} .}
\end{gathered}
$$


Let $e_{\pi}: M \rightarrow M_{N}(A)$ be the function

$$
x \mapsto \sum_{i, j} e_{i j} \otimes \chi_{i}^{1 / 2}(x) \chi_{j}^{1 / 2}(x) v_{i j}(x) .
$$

Because $\psi_{i} \psi_{j}^{-1}(x, a)=\left(x, v_{i j}(x) a\right)$ for $x \in V_{i j}$, it is easy to check that $e_{\pi}(x)$ is the matrix representing the orthogonal projection of $A^{N}$ onto $\theta\left(\left.E_{\pi}\right|_{x}\right)$. In this way we see that $\left[E_{\pi}\right]=\left[e_{\pi}\right] \in K_{0}(C(M) \otimes A)$.

Since $\mathcal{F}=\left\{s_{i j}\right\}$ and $\pi$ is an $(\mathcal{F}, \varepsilon)$-representation, it follows immediately that the transition functions $v_{i j}$ satisfy $\left\|v_{i j}(x)-\pi\left(s_{i j}\right)\right\|<\varepsilon$ for all $x \in V_{i j}$. Thus

$$
\left\|e_{\pi}-(1 \otimes \pi)(e)\right\|=\left\|e_{\pi}-\sum_{i, j} e_{i j} \otimes \chi_{i}^{1 / 2} \chi_{j}^{1 / 2} \pi\left(s_{i j}\right)\right\|<\varepsilon
$$

as well. Recall that $\ell_{\pi}$ is obtained by perturbing $(1 \otimes \pi)(e)$ to a projection using functional calculus and then taking its $K_{0}$-class (see Section 2.2). The previous estimate shows that this class must be $\left[e_{\pi}\right]$.

Remark 4.4. The previous proposition shows that the class $\left[E_{\pi}\right]$ is independent of the order $\mathbf{o}$ on the vertices of $\Lambda_{0}$.

4.5. Connections arising from transition functions. We now define a canonical connection on $E_{\pi}$ associated with the family $\left\{v_{i j}\right\}$ of transition functions. This connection will be used in the proof of Theorem 5.4 .

4.5.1. The smooth sections $\Gamma\left(E_{\pi}\right)$ of $E_{\pi}$ may be identified with

$$
\left\{\left(s_{i}\right) \in \bigoplus_{i} \Omega^{0}\left(V_{i}, E_{\pi}\right): s_{j}=v_{j i} s_{i} \text { on } V_{i j}\right\} .
$$

Let $\nabla_{i}: \Omega^{0}\left(V_{i}, A\right) \rightarrow \Omega^{1}\left(V_{i}, A\right)$ be given by

$$
\nabla_{i}(s)=d s+\omega_{i} s \quad \forall s \in \Omega^{0}\left(V_{i}, A\right),
$$

where

$$
\omega_{i}=\sum_{k} \chi_{k} v_{k i}^{-1} d v_{k i}
$$

Notice that $v_{k i} \in \Omega^{0}\left(V_{i k}, \mathrm{GL}(A)\right)$ and so $\omega_{i}$ may be regarded as an $A$-valued 1-form on $V_{i}$, which can be multiplied fiberwise by the values of the section $s$.

We define a connection $\nabla$ on $E_{\pi}$ by

$$
\nabla\left(s_{i}\right)=\left(\nabla_{i} s_{i}\right) .
$$

That $\nabla$ takes values in $\Omega^{1}\left(M, E_{\pi}\right)$ follows from a straightforward computation verifying

$$
\nabla_{j} s_{j}=v_{j i} \nabla_{i} s_{i}
$$

It is just as straightforward to verify that $\nabla$ is $A$-linear and satisfies the Leibniz rule. 
4.5.2. Define $\Omega_{i}=d \omega_{i}+\omega_{i} \wedge \omega_{i} \in \Omega^{2}\left(V_{i}, A\right)$. One checks that $\Omega_{i}=v_{j i}^{-1} \Omega_{j} v_{j i}$ and so $\left(\Omega_{i}\right)$ defines an element $\Omega$ of $\Omega^{2}\left(M, \operatorname{End}_{A}\left(E_{\pi}\right)\right)$. This is nothing but the curvature of $\nabla$ (see [Sch05, Proposition 3.8]).

\section{The Chern Character}

In this section we prove our main technical result, Theorem 2.6. It computes the trace of the push-forward of $\mu[M]$ in terms of the de la Harpe-Skandalis determinant by using that the cocycle conditions almost hold for the elements $\pi\left(s_{i j}\right)$,

5.1. The de la Harpe-Skandalis determinant. The de la Harpe-Skandalis determinant dlHS84] appears in our formula below. Let us recall the definition. Write $\mathrm{GL}_{\infty}(A)$ for the (algebraic) inductive limit of $\left(\mathrm{GL}_{n}(A)\right)_{n \geq 1}$ with standard inclusions. For a piecewise smooth path $\xi:\left[t_{1}, t_{2}\right] \rightarrow \mathrm{GL}_{\infty}(A)$, define

$$
\widetilde{\Delta}_{\tau}(\xi)=\frac{1}{2 \pi i} \tau\left(\int_{t_{1}}^{t_{2}} \xi^{\prime}(t) \xi(t)^{-1} d t\right)=\frac{1}{2 \pi i} \int_{t_{1}}^{t_{2}} \tau\left(\xi^{\prime}(t) \xi(t)^{-1}\right) d t .
$$

We will make use of some of the properties of $\widetilde{\Delta}_{\tau}$ stated below.

Lemma 5.2 (cf. Lemme 1 of dlHS84]).

(1) Let $\xi_{1}, \xi_{2}:\left[t_{1}, t_{2}\right] \rightarrow \mathrm{GL}_{\infty}^{0}(A)$ be two paths and $\xi$ be their pointwise product. Then $\widetilde{\Delta}_{\tau}(\xi)=\widetilde{\Delta}_{\tau}\left(\xi_{1}\right)+\widetilde{\Delta}_{\tau}\left(\xi_{2}\right)$.

(2) Let $\xi:\left[t_{1}, t_{2}\right] \rightarrow \mathrm{GL}_{\infty}^{0}(A)$ be a path with $\|\xi(t)-1\|<1$ for all $t$. Then

$$
2 \pi i \cdot \widetilde{\Delta}_{\tau}(\xi)=\tau\left(\log \xi\left(t_{2}\right)\right)-\tau\left(\log \xi\left(t_{1}\right)\right) .
$$

(3) The integral $\widetilde{\Delta}_{\tau}(\xi)$ is left invariant under a fixed-end-point homotopy of $\xi$.

5.3. The Chern character on $K_{0}(C(M) \otimes A)$. Assume $\tau$ is a trace on $A$. Then $\tau$ induces a map on $\Omega^{2}\left(V_{i}\right.$, End $\left._{A}\left(\left.E_{\pi}\right|_{V_{i}}\right)\right)$ and by the trace property $\tau\left(\Omega_{i}\right)=\tau\left(\Omega_{j}\right)$ on $V_{i j}$. We obtain in this way a globally defined form $\tau(\Omega) \in \Omega^{2}(M, \mathbb{C})$.

Since the fibers of our bundle are all equal to $A$, and our manifold is 2-dimensional, the definition of the Chern character associated with $\tau$ (from [Sch05, Definition 4.1], but we have included a normalization coefficient) reduces to

$$
\begin{aligned}
& \operatorname{ch}_{\tau}\left(\ell_{\pi}\right)=\tau\left(\exp \left(\frac{i \Omega}{2 \pi}\right)\right)=\tau\left(\sum_{k=0}^{\infty} \frac{i \Omega / 2 \pi \wedge \cdots \wedge i \Omega / 2 \pi}{k !}\right) \\
&=\tau\left(\frac{i \Omega}{2 \pi}\right) \in \Omega^{2}(M, \mathbb{C}) .
\end{aligned}
$$

This is a closed form whose cohomology class does not depend on the choice of the connection $\nabla$ (see Sch05, Lemma 4.2]).

A few remarks are in order before stating the next result.

Because $\Lambda$ is a locally ordered simplicial complex (recall the partial order $\mathbf{o}$ from Section 4.1), every 2-simplex $\sigma$ may be written uniquely as $\left\langle x_{i}, x_{j}, x_{k}\right\rangle$ with the 
vertices written in increasing o-order. Whenever we write a simplex in this way it is implicit that the vertices are written in increasing o-order. We may write $\sigma$ for $\sigma$ along with this order.

The orientation $[M]$ induces an orientation of the boundary of the dual cell $U_{i}$ and in particular of the segment $U_{i k}^{\sigma}$. Let $s(\sigma)=0$ if the initial endpoint of $U_{i k}^{\sigma}$ under this orientation is the barycenter of $\sigma$, and let $s(\sigma)=1$ otherwise.

Theorem 5.4. For a simplex $\sigma=\left\langle x_{i}, x_{j}, x_{k}\right\rangle$ of $\Lambda$, let $\xi_{\sigma}$ be the linear path

$$
\xi_{\sigma}(t)=(1-t) \pi\left(s_{i k}\right)+t \pi\left(s_{i j}\right) \pi\left(s_{j k}\right), \quad t \in[0,1]
$$

in $\operatorname{GL}(A)$. Then

$$
\tau\left(\pi_{\sharp}(\mu[M])\right)=\sum_{\sigma}(-1)^{s(\sigma)} \widetilde{\Delta}_{\tau}\left(\xi_{\sigma}\right),
$$

where the sum ranges over all 2-simplices $\sigma$ of $\Lambda$.

Proof. The path $\xi_{\sigma}$ lies entirely in $\mathrm{GL}(A)$ because $\left\|\pi\left(s_{i k}\right)-\pi\left(s_{i j}\right) \pi\left(s_{j k}\right)\right\|<\varepsilon$. It follows from Theorem 2.4 (on page 4) and Eq. (6) above that

$$
\tau\left(\pi_{\sharp}(\mu[M])\right)=\left\langle\operatorname{ch}_{\tau}\left(\ell_{\pi}\right),[M]\right\rangle=-\frac{1}{2 \pi i} \int_{M} \tau(\Omega) .
$$

We compute this integral.

First observe that by the trace property of $\tau$ we have $\tau\left(\omega_{l} \wedge \omega_{l}\right)=0$ for every $l$. Thus

$$
\begin{gathered}
\int_{M} \tau(\Omega)=\sum_{l} \int_{U_{l}} \tau\left(\Omega_{l}\right)=\sum_{l} \int_{U_{l}} \tau\left(d \omega_{l}+\omega_{l} \wedge \omega_{l}\right)= \\
=\sum_{l} \int_{U_{l}} \tau\left(d \omega_{l}\right)=\sum_{l} \int_{U_{l}} d \tau\left(\omega_{l}\right)=\sum_{l} \int_{\partial U_{l}} \tau\left(\omega_{l}\right),
\end{gathered}
$$

where we used Green's theorem for the last equality and $\partial U_{l}$ has the orientation induced from $[M]$. Recall that $U_{l}$ is the dual cell to $v_{l}$. Write this as a sum over the 2-simplices of $\Lambda$ :

$$
\sum_{l} \int_{\partial U_{l}} \tau\left(\omega_{l}\right)=\sum_{l} \sum_{\sigma} \int_{\left(\partial U_{l}\right) \cap \sigma} \tau\left(\omega_{l}\right)=\sum_{\sigma} \sum_{l} \int_{\left(\partial U_{l}\right) \cap \sigma} \tau\left(\omega_{l}\right) .
$$

Exactly three dual cells meet a 2-simplex $\sigma=\left\langle x_{i}, x_{j}, x_{k}\right\rangle-U_{i}, U_{j}$, and $U_{k}$-so for each simplex there are three integrals we need to account for. Let us treat each of these in turn.

The definition of the connection forms (see Section 4.5.1) implies that $\omega_{i}$ restricted to $\sigma$ equals

$$
\omega_{i}=\chi_{k} v_{k i}^{-1} d v_{k i}+\chi_{j} v_{j i}^{-1} d v_{j i}=\chi_{k} v_{k i}^{-1} d v_{k i},
$$

where the last equality follows from the fact that $v_{j i}$ is constant. Now, $\left(\partial U_{i}\right) \cap \sigma$ is the union of the two segments $U_{i j}^{\sigma}$ and $U_{i k}^{\sigma}$. Observe that $v_{i k}$ is constantly equal to 
$\pi\left(s_{i j}\right) \pi\left(s_{j k}\right)$ on $V_{i} \cap V_{j} \cap V_{k}$ (see Section 4.1.1). Since $U_{i j}^{\sigma} \cap V_{k} \subseteq V_{i} \cap V_{j} \cap V_{k}$ and $\chi_{k}$ vanishes outside $V_{k}$, we get

$$
\int_{\left(\partial U_{i}\right) \cap \sigma} \tau\left(\omega_{i}\right)=\int_{U_{i j}^{\sigma}} \tau\left(\chi_{k} v_{k i}^{-1} d v_{k i}\right)+\int_{U_{i k}^{\sigma}} \tau\left(\chi_{k} v_{k i}^{-1} d v_{k i}\right)=\int_{U_{i k}^{\sigma}} \tau\left(\chi_{k} v_{k i}^{-1} d v_{k i}\right) .
$$

The second integral $\int_{\left(\partial U_{j}\right) \cap \sigma} \tau\left(\omega_{j}\right)$ vanishes. This is because $v_{i j}$ and $v_{j k}$ are constant and so

$$
\omega_{j}=\chi_{i} v_{i j}^{-1} d v_{i j}+\chi_{k} v_{k j}^{-1} d v_{k j}=0 .
$$

The third integral may be calculated just as the first, with the roles of $i$ and $k$ reversed. We obtain

$$
\int_{\left(\partial U_{k}\right) \cap \sigma} \tau\left(\omega_{k}\right)=\int_{U_{k i}^{\sigma}} \tau\left(\chi_{i} v_{i k}^{-1} d v_{i k}\right) .
$$

Combining the three integrals we get

$$
\begin{aligned}
\sum_{\sigma} \sum_{l} \int_{\left(\partial U_{l}\right) \cap \sigma} \tau\left(\omega_{l}\right)=\sum_{\sigma}\left(\int_{U_{i k}^{\sigma}} \tau\left(\chi_{k} v_{k i}^{-1} d v_{k i}\right)+\int_{U_{k i}^{\sigma}} \tau\left(\chi_{i} v_{i k}^{-1} d v_{i k}\right)\right)= \\
=\sum_{\sigma} \int_{U_{i k}^{\sigma}} \tau\left(\chi_{k} v_{k i}^{-1} d v_{k i}-\chi_{i} v_{i k}^{-1} d v_{i k}\right),
\end{aligned}
$$

where the last equality is due to the opposite orientations of the segment $U_{i k}^{\sigma}$ in the preceding two integrals.

It follows from $v_{i k} v_{k i}=1$ that $d v_{i k} v_{i k}^{-1}+v_{k i}^{-1} d v_{k i}=0$. Therefore, the last line in the equation above is equal to

$$
\sum_{\sigma} \int_{U_{i k}^{\sigma}} \tau\left(\chi_{k} v_{k i}^{-1} d v_{k i}+\chi_{i} v_{k i}^{-1} d v_{k i}\right)=\sum_{\sigma} \int_{U_{i k}^{\sigma}} \tau\left(v_{k i}^{-1} d v_{k i}\right)=-\sum_{\sigma} \int_{U_{i k}^{\sigma}} \tau\left(v_{i k}^{-1} d v_{i k}\right) .
$$

To arrive at the conclusion of the theorem, consider the restriction of $v_{i k}$ to the segment $U_{i k}^{\sigma}$. This is the segment between the barycenter of $\sigma$, where $v_{i k}$ takes the value $\pi\left(s_{i j}\right) \pi\left(s_{j k}\right)$, and the barycenter of $\left\langle x_{i}, x_{k}\right\rangle$, where $v_{i k}$ takes the value $\pi\left(s_{i k}\right)$ (see Section 4.1.1). Then

$$
\int_{U_{i k}^{\sigma}} \tau\left(v_{k i}^{-1} d v_{k i}\right)=(-1)^{s(\sigma)} 2 \pi i \cdot \widetilde{\Delta}_{\tau}\left(\xi_{\sigma}\right) .
$$

This concludes the proof.

\section{ORIEnted SURFACES}

For the proof of Theorem 2.6, we will use a convenient triangulation $\Lambda_{g}$ of the orientable genus $g$ surface $\Sigma_{g}$ that we proceed to describe. The covering space of $\Sigma_{g}$ is the open disc and we may take as a fundamental domain a regular $4 g$-gon, call it $\widetilde{\Sigma}_{g}$, drawn in the hyperbolic plane.

Figure 2 depicts a procedure to obtain $\widetilde{\Sigma}_{2}$ by gluing together two copies of $\widetilde{\Sigma}_{1}$. (We will give a more explicit description of $\widetilde{\Sigma}_{g}$ in a moment). It also illustrates the 


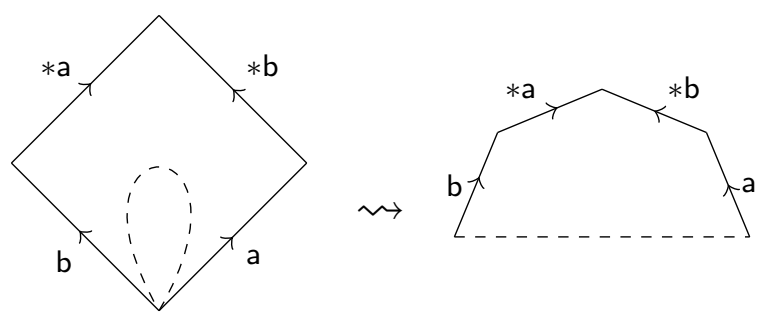

(A)

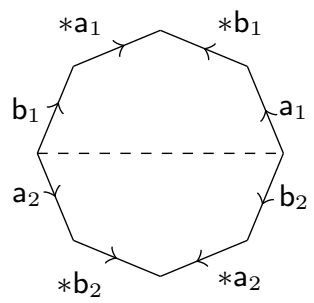

(B)

Figure 2. (A) The fundamental domain $\widetilde{\Sigma}_{1}$ (with a hole). (B) The fundamental domain $\widetilde{\Sigma}_{2}$.

labeling we use for the (oriented) sides of $\widetilde{\Sigma}_{1}$ and $\widetilde{\Sigma}_{2}$. To obtain $\Sigma_{1}$, for example, we identify the side a with $*$ a and the side $\mathrm{b}$ with $*$ b. To obtain the double torus $\Sigma_{2}$, we identify $\mathrm{a}_{k}$ with $* \mathrm{a}_{k}$ and $\mathrm{b}_{k}$ with $* \mathrm{~b}_{k}$ for $k \in\{1,2\}$.

6.1. Triangulations. Let us first define a triangulation $\widetilde{\Lambda}_{g}$ of the fundamental domain $\widetilde{\Sigma}_{g}$. We do this by gluing $g$ triangulated copies of $\widetilde{\Sigma}_{1}$ together. Figure 4 A on page 21 shows the triangulation for the $k$ th copy of $\widetilde{\Sigma}_{1}$ (with a hole), call it $\widetilde{\Lambda}_{1}^{k}$. Ignore the labels on the edges and the highlighted edges for now. The vertex labeling also indicates how to glue $\widetilde{\Lambda}_{1}^{k}$ to $\widetilde{\Lambda}_{1}^{k-1}$ and $\widetilde{\Lambda}_{1}^{k+1}$, with addition modulo $g$. Figure $4 \mathrm{~B}$ illustrates the result of this gluing, the end-result being $\widetilde{\Lambda}_{g}$ by definition.

The underlying space of $\widetilde{\Lambda}_{g}$ is $\widetilde{\Sigma}_{g}$. Identifying all the vertices $v_{i}^{k}$, as well as identifying $a_{i}^{k}$ with $* a_{i}^{k}$ and $b_{i}^{k}$ with $* b_{i}^{k}$, for each $i \in\{1,2\}$ and $k \in\{1, \ldots, n\}$, yields a triangulation $\Lambda_{g}$ of $\Sigma_{g}$.

6.2. Surface groups. We identify the fundamental group $\Gamma_{g}$ of $\Sigma_{g}$ with the group of deck transformations of the universal covering space of $\Sigma_{g}$. We give a more concrete description of this group now.

The fundamental domain $\widetilde{\Sigma}_{g}$ is a regular $4 g$-gon. We write $\mathrm{a}_{k}, \mathrm{~b}_{k} * \mathrm{a}_{k}$ and $* \mathrm{~b}_{k}$, $k \in\{1, \ldots, n\}$, for its (oriented) sides. The triangulation $\widetilde{\Lambda}_{g}$ gives a subdivision of the side $\mathrm{a}_{k}$ into the three edges in the path $\left(v_{0}^{k}, a_{1}^{k}, a_{2}^{k}, v_{1}^{k}\right)$ (with orientation given by the directed edge $\left.\left(a_{1}^{k}, a_{2}^{k}\right)\right)$. The subdivision of the sides $\mathrm{b}_{k}, * \mathrm{a}_{k}$ and $* \mathrm{~b}_{k}$ is similar. See Figure 4 A.

The group of deck transformations $\Gamma_{g}$ is generated by the hyperbolic isometries $\alpha_{k}$ and $\beta_{k}, k \in\{1, \ldots, g\}$, defined as follows: $\alpha_{k}$ maps $* \mathrm{a}_{k}$ to $\mathrm{a}_{k}$ in such a way that, locally, the half-plane bounded by $* a_{k}$ containing $\widetilde{\Sigma}_{g}$ is mapped to the halfplane bounded by $\mathrm{a}_{k}$ but opposite $\widetilde{\Sigma}_{g}$. The transformation $\beta_{k}$ is defined analogously, mapping $* \mathrm{~b}_{k}$ to $\mathrm{b}_{k}$. We refer the reader to [ve92, Chapter VII] for more details. When $g=1$, for example, the transformations $\alpha_{1}$ and $\beta_{1}$ are just translations. See 
Figure 3, where we have omitted the sub- and superscripts corresponding to $k=1$, since $g=1$.

For $k \in\{1, \ldots, g\}$, let

$$
\kappa_{k}=\prod_{j=1}^{k}\left[\alpha_{k}, \beta_{k}\right]
$$

and let $\kappa_{0}=1$. We have that $\kappa_{g}=1$.

6.3. Local orders and trees. We need $\Lambda_{g}$ to be locally ordered, so we proceed to fix a partial order on the vertices of $\Lambda_{g}$ such that the vertices of every simplex form a totally ordered set. Let us define an order on the vertices of $\widetilde{\Lambda}_{g}$ that drops down to the order we need. On the $k$ th copy $\widetilde{\Lambda}_{1}^{k}$, the corresponding order is indicated in Figure $4 \mathrm{~A}$ by arrows on the edges, always pointing from a smaller vertex to a larger one. It is defined as follows:

- for the "inner" vertices we go "counter-clockwise": for fixed $k \in\{1, \ldots, g\}$, $w_{i}^{k}<w_{j}^{k}$ if $i<j$, except when $k=g$ and $j=4$ (in which case $w_{4}^{g}=w_{0}^{1}$ and we already have $\left.w_{0}^{1}<w_{k}^{i}\right)$;

- the "inner" vertices are larger than the "outer" ones: $w_{i}^{k}>v_{j}^{l}, a_{j}^{l}, b_{j}^{l}, * a_{j}^{l}$, $* b_{j}^{l}$ for all $i, j, k$ and $l$;

- for the "outer" vertices: $v_{i}^{k}<a_{j}^{l}, b_{j}^{l}, * a_{j}^{l}, * b_{j}^{l}$ for all $i, j, k$ and $l$; for every $k$, $a_{1}^{k}<a_{2}^{k}, * a_{1}^{k}<* a_{2}^{k}$, and similarly for the $b_{j}^{k}$.

Finally, we will need a spanning tree $T_{g}$ of $\Lambda_{g}$, and a lift $\widetilde{T}_{g}$ to the triangulation $\widetilde{\Lambda}_{g}$ of the fundamental domain $\widetilde{\Sigma}_{g}$. Again, we define $\widetilde{T}_{g}$ first. It is obtained as the union of the edge between $w_{0}^{1}$ and $v_{0}^{1}$ (including those two vertices) and trees in each copy $\Sigma_{1}^{k}$. The tree in $\Sigma_{1}^{k}$ is depicted in Figure 44 by highlighted (heavier) edges. This drops to a spanning tree $T_{g}$ of $\Sigma_{g}$. We regard $T_{g}$ as "rooted" at the vertex $v_{0}^{1}$. (In the notation of 3.1 , where the vertices were labeled consecutively as $x_{0}, \ldots, x_{N}$, we have that $v_{0}^{1}=x_{0}$.)

\section{Proof of the MAIN ReSUlt}

This section contains the proof of Theorem 2.6. The proof is split into a number of lemmas.

To apply Theorem 5.4 we will first compute the group element $s_{i j}$ corresponding to each edge $x_{i} x_{j}$ of $\Lambda_{g}$, in the sense discussed in Section 3.1. Equivalently, we compute group elements corresponding to edges in the cover $\Lambda_{g}$, keeping in mind that the lifts of any edge of $\Lambda_{g}$ will all correspond to the same group element.

A concise way of stating the result of these computations is to label each edge in Figure 44 with the corresponding group element.

Lemma 7.1. The labels in Figure $4 \mathrm{~A}$ are correct.

Proof. We carry out the computations in three separate claims. 
Claim. An edge of the form $a_{i}^{k} w_{j}^{k}$ corresponds to $\alpha_{k}^{-1} \in \Gamma_{g}$. Similarly, an edge of the form $b_{i}^{k} w_{j}^{k}$ corresponds to $\beta_{k}^{-1} \in \Gamma_{g}$.

Consider $a_{i}^{k} w_{j}^{k}$ first. When we add this edge to the forest that is the union of all the lifts of $T_{g}$ (that is, translates of $\widetilde{T}_{g}$ ), we obtain a unique path $P$ between $v_{0}^{1}$, our root vertex, and some translate $s v_{0}^{1}$, where $s \in \Gamma_{g}$. We regard $P$ as directed in the direction of the edge $a_{i}^{k} w_{j}^{k}$ that we started with, so it is a path from $s v_{0}^{1}$ to $v_{0}^{1}$. It therefore drops down to a loop in $\Sigma_{g}$ whose class is $s^{-1}$, the group element we want to compute (see Hat02, Proposition 1.39], for example). Now notice that because $* a_{i}^{k}$ belongs to $\widetilde{T}_{g}$, its translate $\alpha_{k}\left(* a_{i}^{k}\right)=a_{i}^{k}$ belongs to the translate $\alpha_{k} \widetilde{T}_{g}$ of $\widetilde{T}_{g}$. Thus $P$ is a path between $v_{0}^{k}$ and $\alpha_{k} v_{0}^{k}$. The corresponding group element is therefore $\alpha_{k}^{-1}$. An entirely similar argument applies to the edge $b_{i}^{k} w_{j}^{k}$.

Claim. Any edge between inner vertices (vertices of the form $w_{i}^{k}$ ) corresponds to $1 \in \Gamma_{g}$. The edges $a_{1}^{k} a_{2}^{k}, b_{1}^{k} b_{2}^{k}, * a_{1}^{k} * a_{2}^{k}$, and $* b_{1}^{k} * b_{2}^{k}$ all correspond to $1 \in \Gamma_{g}$.

We proceed as in the previous claim. Any edge between inner vertices is either in $\widetilde{T}_{g}$ or between two vertices that are in $\widetilde{T}_{g}$. The associated path we get is therefore from $v_{0}^{k}$ to itself. The same is true of the edges $b_{1}^{k} b_{2}^{k}$ and $* a_{1}^{k} * a_{2}^{k}$. It follows that the corresponding group element is 1 . Since $a_{1}^{k} a_{2}^{k}$ and $* a_{1}^{k} * a_{2}^{k}$ are both lifts of the same edge, they correspond to the same element. Similarly, $* b_{1}^{k} * b_{2}^{k}$ corresponds to 1.

Claim. An edge that is incident to $v_{i}^{k}$ and to a vertex $z$ in the tree $\widetilde{T}_{g}$ corresponds to the element $s \in \Gamma_{g}$ such that $v_{0}^{1}=s v_{i}^{k}$. (The edge is given the orientation induced by the order on the vertices, as usual.) For $k \in\{1, \ldots, g\}$,

$$
\begin{aligned}
& v_{0}^{1}=\kappa_{k-1} \cdot v_{0}^{k} \\
& v_{0}^{1}=\kappa_{k-1} \alpha_{k} \beta_{k} \alpha_{k}^{-1} \cdot v_{1}^{k} \\
& v_{0}^{1}=\kappa_{k-1} \alpha_{k} \beta_{k} \cdot v_{2}^{k} \\
& v_{0}^{1}=\kappa_{k-1} \alpha_{k} \cdot v_{3}^{k}
\end{aligned}
$$

(Recall that $\kappa_{k}$ is the product of commutators $\left[\alpha_{1}, \beta_{1}\right]\left[\alpha_{2}, \beta_{2}\right] \cdots\left[\alpha_{k}, \beta_{k}\right]$ for $k \in$ $\{1, \ldots, g\}$, and that $\kappa_{0}=1$.)

Observe that, because of how the order was defined, $v_{i}^{k}<z$ always holds. When we add the edge $v_{i}^{k} z$ to the tree $\widetilde{T}_{g}$ we obtain a path from $v_{i}^{k}$ to $v_{0}^{1}$. (See Figure 4 . but keep in mind that in the case $k=1$ the edge $v_{0}^{1} w_{0}^{1}$ belongs to the tree.) It follows that the corresponding element is the $s \in \Gamma_{g}$ such that $v_{0}^{1}=s v_{i}^{k}$.

To compute these elements $s$ we argue by induction on $k$. Assume $k=1$. We observe that

$$
v_{4}^{1} \stackrel{\beta_{1}^{-1}}{\longmapsto} v_{1}^{1} \stackrel{\alpha_{1}^{-1}}{\longmapsto} v_{2}^{1} \stackrel{\beta_{1}}{\longmapsto} v_{3}^{1} \stackrel{\alpha_{1}}{\longmapsto} v_{0}^{1} .
$$


Indeed, from the definition (see Section 6.2) we see that the transformation $\alpha_{1}$ takes $v_{3}^{1}$ to $v_{0}^{1}$ - think of the side $* \mathrm{a}_{1}=\left(v_{3}^{1}, * a_{1}^{1}, * a_{2}^{1}, v_{2}^{1}\right)$ being mapped to the side $\mathrm{a}_{1}=\left(v_{0}^{1}, a_{1}^{1}, a_{2}^{1}, v_{1}^{1}\right)$ : the vertex $* a_{1}^{1}$ is mapped to $a_{1}^{1}$ and so $v_{3}^{1}$ is mapped to $v_{0}^{1}$. We also see from Section 6.2 and Figure 44 that $\beta_{1}$ maps $v_{2}^{1}$ to $v_{3}^{1}$, and so $v_{0}^{1}=\alpha_{1} \beta_{1} \cdot v_{2}^{1}$. A similar argument shows that $v_{0}^{1}=\alpha_{1} \beta_{1} \alpha_{1}^{-1} \cdot v_{1}^{1}$ and that

$$
v_{0}^{1}=\alpha_{1} \beta_{1} \alpha_{1}^{-1} \beta_{1}^{-1} \cdot v_{4}^{1}=\kappa_{1} \cdot v_{4}^{1} .
$$

Assuming the computations hold for $k-1$, we prove them for $k$. In fact, most of the work is already done. The same argument we used for the case $k=1$ shows that

$$
v_{4}^{k} \stackrel{\beta_{k}^{-1}}{\longmapsto} v_{1}^{k} \stackrel{\alpha_{k}^{-1}}{\longmapsto} v_{2}^{k} \stackrel{\beta_{k}}{\longmapsto} v_{3}^{k} \stackrel{\alpha_{k}}{\longmapsto} v_{0}^{k} .
$$

The inductive hypothesis implies that

$$
\kappa_{k-1} v_{0}^{k}=\kappa_{k-1} v_{4}^{k-1}=v_{0}^{1},
$$

This ends the proof of the claim.

These three claims prove that the labels in Figure $4 \mathrm{~A}$ are correct.

(The labels in Figure 3 also follow from these calculations, but may be obtained by more straightforward arguments because the generators of $\Gamma_{1} \cong \mathbb{Z}^{2}$ may be regarded as shifts in the plane.)

Notation 7.2. For $k \in\{1, \ldots, g\}$, let

$$
\mathcal{F}_{k}=\left\{\alpha_{k}^{-1}, \beta_{k}^{-1}, \kappa_{k-1}, \kappa_{k-1} \alpha_{k}, \kappa_{k-1} \alpha_{k} \beta_{k}, \kappa_{k-1} \alpha_{k} \beta_{k} \alpha_{k}^{-1}\right\} .
$$

Notice that the set $\mathcal{F}=\left\{s_{i j}\right\}$ considered in Section 3.1 is equal to the union $\mathcal{F}_{1} \cup$ $\mathcal{F}_{1}^{-1} \cup \cdots \cup \mathcal{F}_{g} \cup \mathcal{F}_{g}^{-1}$ by Lemma 7.1 .

7.3. Choosing quasi-representations. We want to apply Theorem 5.4 using the labels obtained in Lemma 7.1 and some convenient choice of a quasi-representation of $\Gamma_{g}$ in $U(A)$. We begin by proving a slightly stronger version Proposition 2.8, which guarantees the existence of quasi-representations (under certain conditions). Let us set up some notation first.

For certain unitaries $u_{1}, v_{1}, \ldots, u_{g}, v_{g}$ in $A$ we will need to produce a quasirepresentation $\pi$ satisfying

$$
\pi\left(\alpha_{k}\right)=u_{k}, \text { and } \pi\left(\beta_{k}\right)=v_{k} \quad \forall k \in\{1, \ldots, g\} .
$$

Write $\mathbb{F}_{2 g}=\left\langle\hat{\alpha}_{1}, \hat{\beta}_{1}, \ldots, \hat{\alpha}_{g}, \hat{\beta}_{g}\right\rangle$ for the free group on $2 g$ generators. Let $q: \mathbb{F}_{2 g} \rightarrow \Gamma_{g}$ and $\hat{\pi}: \mathbb{F}_{2 g} \rightarrow U(A)$ be the homomorphisms given by

$$
q\left(\hat{\alpha}_{k}\right)=\alpha_{k}, \quad q\left(\hat{\beta}_{k}\right)=\beta_{k}
$$

and

$$
\hat{\pi}\left(\hat{\alpha}_{k}\right)=u_{k}, \quad \hat{\pi}\left(\hat{\beta}_{k}\right)=v_{k}
$$




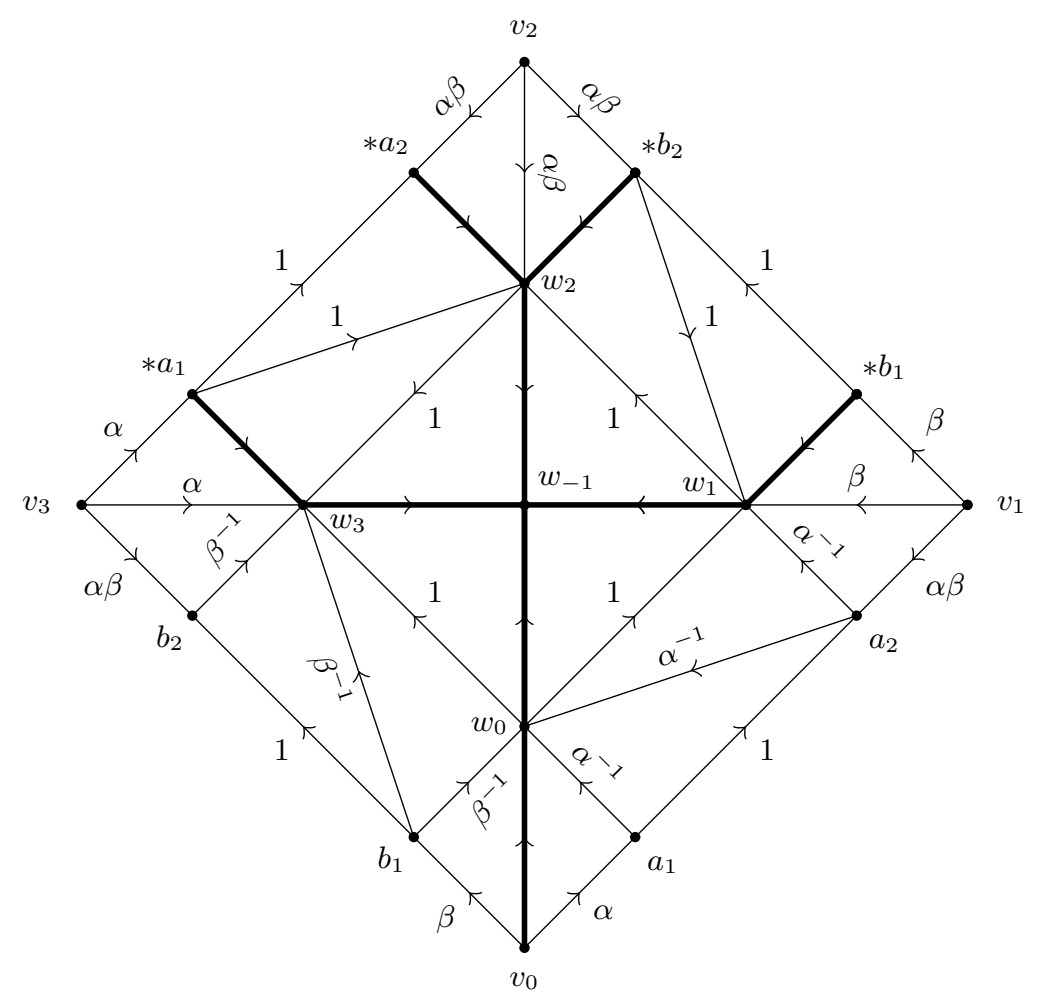

Figure 3 . The triangulation $\widetilde{\Lambda}_{1}$ of $\widetilde{\Sigma}_{1}$. Edges are labeled with the group element associated with the loop they induce. The spanning tree $T_{1}$ of $\Lambda_{1}$ (heavier edges) is "rooted" at $v_{0}$.

for all $k \in\{1, \ldots, g\}$. Notice that the kernel of $q$ is the normal subgroup generated by

$$
\hat{\kappa}_{g}:=\prod_{k=1}^{g}\left[\hat{\alpha}_{k}, \hat{\beta}_{k}\right]
$$

and therefore consists of products of elements of the form $\hat{\gamma} \hat{\kappa}_{g}^{ \pm 1} \hat{\gamma}^{-1}$ where $\hat{\gamma} \in \mathbb{F}_{2 g}$.

Choose a set-theoretic section $s: \Gamma_{g} \rightarrow \mathbb{F}_{2 g}$ of $q$ such that $s(1)=1$,

$$
s\left(\alpha_{k}\right)=\hat{\alpha}_{k}, \quad \text { and } \quad s\left(\beta_{k}\right)=\hat{\beta}_{k} \quad \forall k \in\{1, \ldots, g\} .
$$

Lemma 7.4. For all $\varepsilon>0$ there exists $\delta(\varepsilon)>0$ such that if $A$ is a unital $C^{*}$-algebra and $u_{1}, v_{1}, \ldots, u_{g}, v_{g} \in U(A)$ satisfy

$$
\left\|\prod_{i=1}^{g}\left[u_{i}, v_{i}\right]-1\right\|<\delta(\varepsilon),
$$




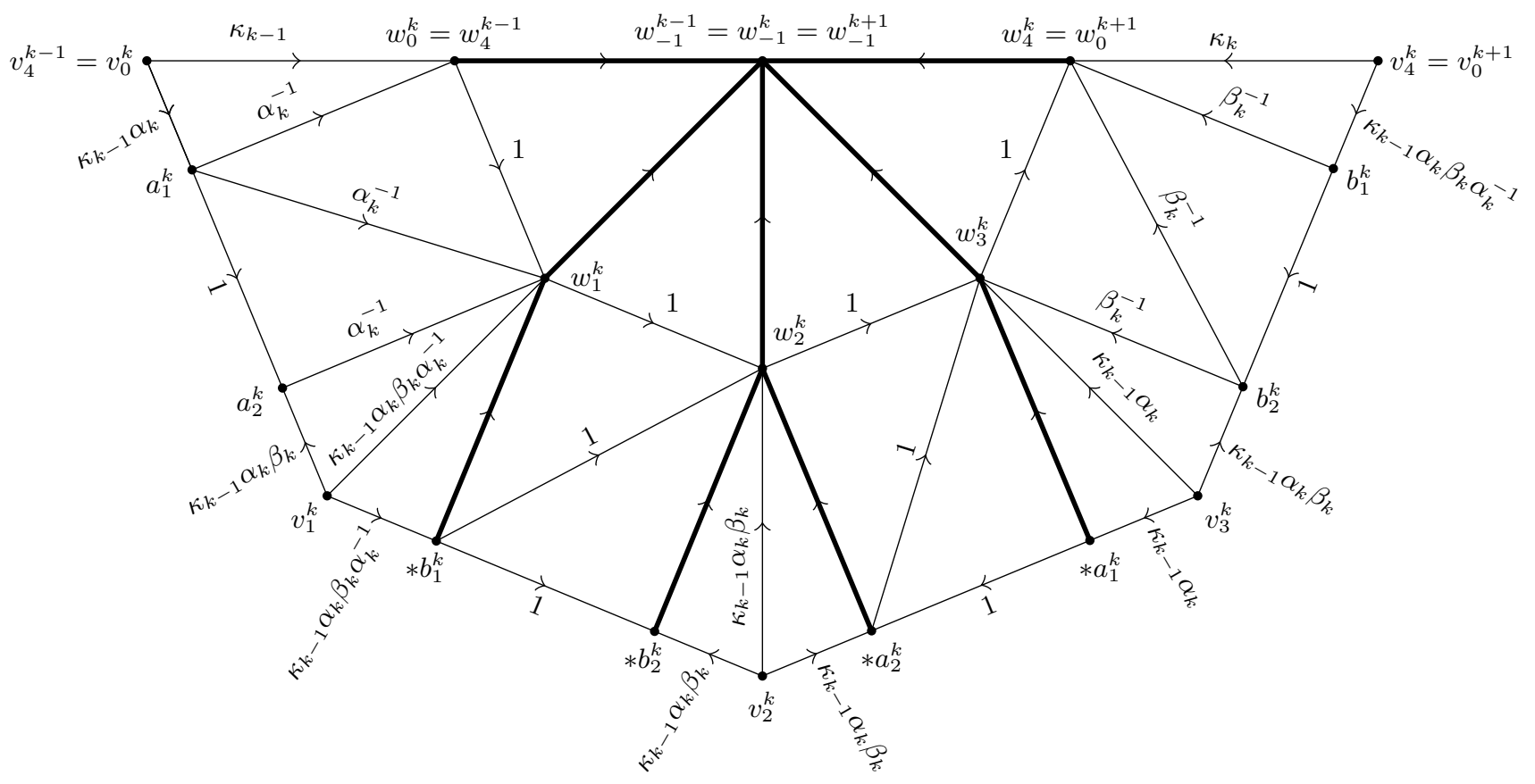

(A)

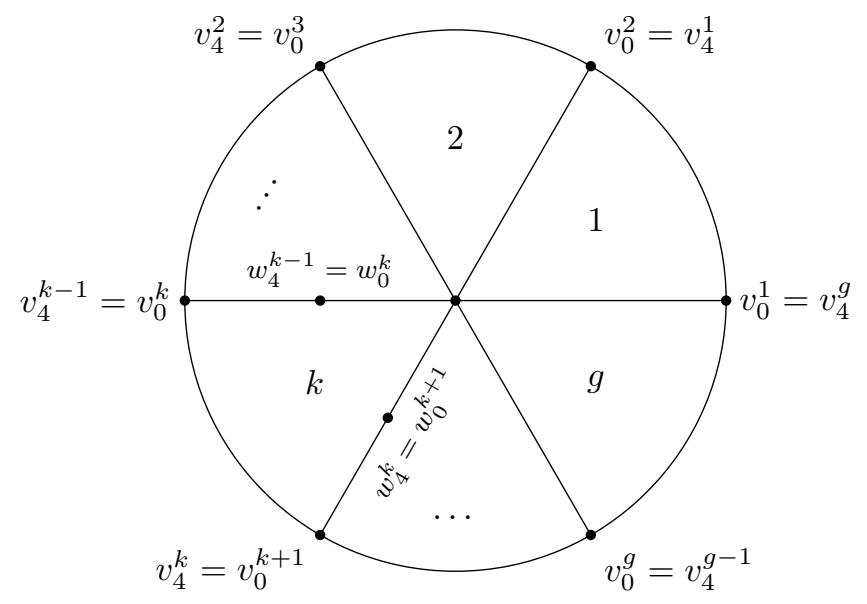

(B)

FiguRE 4. (A) The triangulation we use for $\widetilde{\Sigma}_{1}^{k}$, the $k$ th copy of $\widetilde{\Sigma}_{1}$ (with a hole). Every edge is labeled with the element of $\Gamma_{g}$ corresponding to the loop it induces. (B) How the simplicial complex $\widetilde{\Sigma}_{g}$ is defined. The $k$ th "wedge" is pictured in (A). 
then $\pi=\hat{\pi} \circ s$ (with $s$ as constructed above) is an $(\mathcal{F}, \varepsilon)$-representation satisfying Eq. (7).

This lemma obviously implies Proposition 2.8 .

Proof. We only need to check that $\pi$ is $(\mathcal{F}, \varepsilon)$-multiplicative. Assume that Eq. (8) holds for some $\delta$ in place of $\delta(\varepsilon)$.

Because $\hat{\pi}$ is a homomorphism, for all $\gamma, \gamma^{\prime} \in \Gamma_{g}$ we have

$$
\left\|\pi(\gamma) \pi\left(\gamma^{\prime}\right)-\pi\left(\gamma \gamma^{\prime}\right)\right\|=\left\|\pi(\gamma) \pi\left(\gamma^{\prime}\right) \pi\left(\gamma \gamma^{\prime}\right)^{*}-1\right\|=\left\|\hat{\pi}\left(s(\gamma) s\left(\gamma^{\prime}\right) s\left(\gamma \gamma^{\prime}\right)^{-1}\right)-1\right\| .
$$

Now, $s(\gamma) s\left(\gamma^{\prime}\right) s\left(\gamma \gamma^{\prime}\right)^{-1}$ is in the kernel of $q$ and is therefore a product of the form

$$
\prod_{i=1}^{m} \hat{\gamma}_{i} \hat{\kappa}_{g}^{\varepsilon_{i}} \hat{\gamma}_{i}^{-1}
$$

where $m$ depends on $\gamma$ and $\gamma^{\prime}$ and $\varepsilon_{i} \in\{1,-1\}$. Thus

$$
\begin{aligned}
\left\|\pi(\gamma) \pi\left(\gamma^{\prime}\right)-\pi\left(\gamma \gamma^{\prime}\right)\right\| & =\left\|\hat{\pi}\left(\prod_{i=1}^{m} \hat{\gamma}_{i} \hat{\kappa}_{g}^{\varepsilon_{i}} \hat{\gamma}_{i}^{-1}\right)-1\right\| \\
& \leq \sum_{i=1}^{m}\left\|\hat{\pi}\left(\hat{\gamma}_{i}\right) \hat{\pi}\left(\hat{\kappa}_{g}\right)^{\varepsilon_{i}} \hat{\pi}\left(\hat{\gamma}_{i}\right)^{*}-1\right\| \\
& \leq m\left\|\prod_{i=1}^{g}\left[u_{i}, v_{i}\right]-1\right\|<m \delta .
\end{aligned}
$$

Since $\mathcal{F}$ is a finite set, there is a positive integer $M$ such that if $\gamma, \gamma^{\prime} \in \mathcal{F}$, then $s(\gamma) s\left(\gamma^{\prime}\right) s\left(\gamma \gamma^{\prime}\right)^{-1}$ is a product of at most $M$ elements of the form $\hat{\gamma}_{i} \hat{\kappa}_{g}^{\varepsilon_{i}} \hat{\gamma}_{i}^{-1}$ as above. It follows that $\pi$ is an $(\mathcal{F}, M \delta)$-representation. Choose $\delta(\varepsilon)=\varepsilon / M$.

Notation 7.5. Recall the set $\mathcal{F}_{k}$ defined in Notation 7.2. Let $s_{0}: \Gamma_{g} \rightarrow \mathbb{F}_{2 g}$ be a set-theoretic section of $q$ such that

$$
\begin{gathered}
s_{0}\left(\alpha_{k}^{ \pm 1}\right)=\hat{\alpha}_{k}^{ \pm 1}, \quad s_{0}\left(\beta_{k}^{ \pm 1}\right)=\hat{\beta}_{k}^{ \pm 1}, \quad s_{0}\left(\kappa_{k-1}\right)=\hat{\kappa}_{k-1}, \\
s_{0}\left(\kappa_{k-1} \alpha_{k}\right)=\hat{\kappa}_{k-1} \hat{\alpha}_{k}, \quad s_{0}\left(\kappa_{k-1} \alpha_{k} \beta_{k}\right)=\hat{\kappa}_{k-1} \hat{\alpha}_{k} \hat{\beta}_{k}
\end{gathered}
$$

for all $k \in\{1, \ldots, g\}$, and

$$
s_{0}\left(\kappa_{k-1} \alpha_{k} \beta_{k} \alpha_{k}^{-1}\right)=\hat{\kappa}_{k-1} \hat{\alpha}_{k} \hat{\beta}_{k} \hat{\alpha}_{k}^{-1}
$$

for all $k \in\{1, \ldots, g-1\}$. That such a section exists follows from the fact that all the words in the list $\mathcal{F}_{1} \cup \cdots \cup \mathcal{F}_{g} \cup\left\{\alpha_{1}, \beta_{1}, \ldots \alpha_{g}, \beta_{g}\right\}$ are distinct, with two exceptions: $\alpha_{1}=\kappa_{0} \alpha_{1} \in \mathcal{F}_{1}$ appears twice, as does $\beta_{g}=\kappa_{g-1} \alpha_{g} \beta_{g} \alpha_{g}^{-1} \in \mathcal{F}_{g}$.

Define $\pi_{0}=\hat{\pi} \circ s_{0}: \Gamma_{g} \rightarrow U(A)$.

Lemma 7.6. If $\left\langle x_{i}, x_{j}, x_{k}\right\rangle$ is any 2-simplex in $\Lambda_{g}$ different from $\left\langle v_{1}^{g}, a_{2}^{g}, w_{1}^{g}\right\rangle$, then $\pi_{0}\left(s_{i k}\right)=\pi_{0}\left(s_{i j}\right) \pi_{0}\left(s_{j k}\right)$. 


$$
\begin{aligned}
& \text { If }\left\langle x_{i}, x_{j}, x_{k}\right\rangle=\left\langle v_{1}^{g}, a_{2}^{g}, w_{1}^{g}\right\rangle \text {, then } \pi_{0}\left(s_{i k}\right)=v_{g} \text { and } \\
& \qquad \pi_{0}\left(s_{i j}\right) \pi_{0}\left(s_{j k}\right)=\left(\prod_{i=1}^{g}\left[u_{i}, v_{i}\right]\right) v_{g} .
\end{aligned}
$$

Proof. The definition of $s_{0}$ implies that the image under $s_{0}$ of any "word" in the list $\mathcal{F}_{k}$ is the word obtained by replacing $\alpha_{k}^{ \pm 1}$ by $\hat{\alpha}_{k}^{ \pm 1}$ and $\beta_{k}^{ \pm 1}$ by $\hat{\beta}_{k}^{ \pm 1}$, with one exception: the image of $\kappa_{g-1} \alpha_{g} \beta_{g} \alpha_{g}^{-1}=\beta_{g}$ under $s_{0}$ is $\hat{\beta}_{g}$.

This observation along with inspection of Figure 4 A shows that $s_{0}\left(s_{i k}\right)=s_{0}\left(s_{i j}\right) s_{0}\left(s_{j k}\right)$ for every 2-simplex in $\Lambda_{g}$ different from $\left\langle v_{1}^{g}, a_{2}^{g}, w_{1}^{g}\right\rangle$. For instance, let $l \in\{1, \ldots, g\}$ and consider the simplex

$$
\left\langle v_{0}^{l}, a_{1}^{l}, w_{0}^{l}\right\rangle=\left\langle x_{i}, x_{j}, x_{k}\right\rangle
$$

The corresponding group elements are

$$
\begin{aligned}
s_{i j} & =\kappa_{l-1} \alpha_{l} \\
s_{j k} & =\alpha_{l}^{-1}, \quad \text { and } \\
s_{i k} & =\kappa_{l-1} .
\end{aligned}
$$

Then

$$
s_{0}\left(s_{i k}\right)=\hat{\kappa}_{l-1}=\hat{\kappa}_{l-1} \hat{\alpha}_{l} \cdot \hat{\alpha}_{l}^{-1}=s_{0}\left(\kappa_{l-1} \alpha_{l}\right) \cdot s_{0}\left(\alpha_{l}^{-1}\right)=s_{0}\left(s_{i j}\right) \cdot s_{0}\left(s_{j k}\right) .
$$

The computations in all other 2 -simplices but $\left\langle v_{1}^{g}, a_{2}^{g}, w_{1}^{g}\right\rangle$ are very similar. For this exceptional simplex we get

$$
s_{0}\left(s_{i k}\right)=s_{0}\left(\kappa_{g-1} \alpha_{g} \beta_{g} \alpha_{g}^{-1}\right)=s_{0}\left(\beta_{g}\right)=\hat{\beta}_{g}
$$

but

$$
s_{0}\left(s_{i j}\right) s_{0}\left(s_{j k}\right)=s_{0}\left(\kappa_{g-1} \alpha_{g} \beta_{g}\right) s_{0}\left(\alpha_{g}^{-1}\right)=\hat{\kappa}_{g-1} \hat{\alpha}_{g} \hat{\beta}_{g} \hat{\alpha}_{g}^{-1}=\hat{\kappa}_{g} \hat{\beta}_{g}
$$

Since $\pi_{0}=\hat{\pi} \circ s_{0}$ and $\hat{\pi}$ is a homomorphism, the lemma follows.

Recall that we used Theorem 2.4 to define $\omega>0$ in Section 3.4 .

Lemma 7.7. If $0<\varepsilon<\omega$ and Eq. (8) holds (so that $\pi_{0}$ is an $(\mathcal{F}, \varepsilon)$-representation), then

$$
\tau\left(\pi_{0 \sharp}\left(\mu\left[\Sigma_{g}\right]\right)\right)=\frac{1}{2 \pi i} \tau\left(\log \left(\prod_{i=1}^{g}\left[u_{i}, v_{i}\right]\right)\right) .
$$

Proof. We apply Theorem 5.4. For each simplex $\left\langle x_{i}, x_{j}, x_{k}\right\rangle$ we compute $\widetilde{\Delta}_{\tau}(\xi)$ where $\xi_{\sigma}$ is the path

$$
\xi_{\sigma}(t)=(1-t) \pi\left(s_{i k}\right)+t \pi\left(s_{i j}\right) \pi\left(s_{j k}\right), \quad t \in[0,1] .
$$

Observe that the value of $\widetilde{\Delta}_{\tau}$ on a constant path is 0 . Lemma 7.6 implies that there is only one 2-simplex $\sigma$ such that $\xi_{\sigma}$ is not constant: $\sigma_{0}=\left\langle v_{1}^{g}, a_{2}^{g}, w_{1}^{g}\right\rangle$. By 
Lemma 7.6 it yields the linear path $\xi_{\sigma_{0}}$ from $v_{g}$ to

$$
\left(\prod_{i=1}^{g}\left[u_{i}, v_{i}\right]\right) v_{g}
$$

Using Lemma 5.2 we obtain

$$
\widetilde{\Delta}_{\tau}\left(\xi_{\sigma_{0}}\right)=\frac{1}{2 \pi i} \tau\left(\log \left(\prod_{i=1}^{g}\left[u_{i}, v_{i}\right]\right)\right) .
$$

Finally, Theorem 5.4 implies

$$
\tau\left(\pi_{0 \sharp}\left(\mu\left[\Sigma_{g}\right]\right)\right)=(-1)^{s\left(\sigma_{0}\right)} \frac{1}{2 \pi i} \tau\left(\log \left(\prod_{i=1}^{g}\left[u_{i}, v_{i}\right]\right)\right)
$$

where the sign $(-1)^{s\left(\sigma_{0}\right)}$ depends on the the orientation $\left[\Sigma_{g}\right]$. The standard orientation on $\Sigma_{g}$ gives $s\left(\sigma_{0}\right)=1$.

By putting these lemmas together we can prove Theorem 2.6.

Proof of Theorem 2.6. Recall that the statement of the theorem fixes a positive integer $g$ and idempotents $q_{0}$ and $q_{1}$ in some matrix algebra over $\ell^{1}\left(\Gamma_{g}\right)$ such that $\mu\left[\Sigma_{g}\right]=\left[q_{0}\right]-\left[q_{1}\right] \in K_{0}\left(\ell^{1}\left(\Gamma_{g}\right)\right)$.

Let $\mathcal{F}_{0}$ be the finite set $\left\{s_{i j}\right\}$ defined in Section 3.1 and described explicitly in Notation 7.2. Theorem 2.4 provides an $\omega>0$ so small that if $\pi: \Gamma_{g} \rightarrow U(A)$ is an $\left(\mathcal{F}_{0}, \omega\right)$-representation, then $\pi_{\sharp}\left(\mu\left[\Sigma_{g}\right]\right):=\pi_{\sharp}\left(q_{0}\right)-\pi_{\sharp}\left(q_{1}\right)$ is defined and

$$
\tau\left(\pi_{\sharp}\left(\mu\left[\Sigma_{g}\right]\right)\right)=\left\langle\operatorname{ch}_{\tau}\left(\ell_{\pi}\right),\left[\Sigma_{g}\right]\right\rangle .
$$

By setting $u_{i}:=\pi\left(\alpha_{i}\right)$ and $v_{i}:=\pi\left(\beta_{i}\right)$ for all $i \in\{1, \ldots, g\}$, we see that such a quasi-representation $\pi$ may be used to define a quasi-representation $\pi_{0}$ as in Section 7.5. The more multiplicative $\pi$ is on $\mathcal{F}_{0}$, the smaller the quantity

$$
\left\|\prod_{i=1}^{g}\left[u_{i}, v_{i}\right]-1\right\|
$$

is. Lemma 7.4 shows that by making this quantity smaller we can make $\pi_{0}$ more multiplicative on $\mathcal{F}_{0}$. Therefore, because $\pi$ and $\pi_{0}$ agree on the generators of $\Gamma_{g}$, there exists an $0<\varepsilon_{0}<\omega$ so small that if $\pi$ is an $\left(\mathcal{F}_{0}, \varepsilon_{0}\right)$-representation, then $\pi_{\sharp}$ and $\pi_{0 \sharp}$ agree on $\left\{q_{0}, q_{1}\right\} \subset K_{0}\left(\ell^{1}\left(\Gamma_{g}\right)\right)$.

Finally,

$$
\tau\left(\pi_{\sharp}\left(\mu\left[\Sigma_{g}\right]\right)\right)=\tau\left(\pi_{0 \sharp}\left(\mu\left[\Sigma_{g}\right]\right)\right)=\frac{1}{2 \pi i} \tau\left(\log \left(\prod_{i=1}^{g}\left[u_{i}, v_{i}\right]\right)\right)
$$

by Lemma 7.7 . 


\section{REFERENCES}

[BMV05] Hela Bettaieb, Michel Matthey, and Alain Valette, Unbounded symmetric operators in Khomology and the Baum-Connes conjecture, J. Funct. Anal. 229 (2005), no. 1, 184-237. MR 2180079

[CGM90] Alain Connes, Mikhaïl Gromov, and Henri Moscovici, Conjecture de Novikov et fibrés presque plats, C. R. Acad. Sci. Paris Sér. I Math. 310 (1990), no. 5, 273-277. MR 1042862

[Dad11] Marius Dadarlat, Group quasi-representations and almost-flat bundles, to appear in J. Noncommutative Geometry, 2011.

[Dad12] _ Group quasi-representations and index theory, J. Topol. Anal. 4 (2012), no. 3, 297-319. MR 2982445

[dlHS84] Pierre de la Harpe and Georges Skandalis, Déterminant associé à une trace sur une algébre de Banach, Ann. Inst. Fourier (Grenoble) 34 (1984), no. 1, 241-260. MR 743629

[EL91] Ruy Exel and Terry A. Loring, Invariants of almost commuting unitaries, J. Funct. Anal. 95 (1991), no. 2, 364-376. MR 1092131

[Exe93] Ruy Exel, The soft torus and applications to almost commuting matrices, Pacific J. Math. 160 (1993), no. 2, 207-217. MR 1233352

[Hat02] Allen Hatcher, Algebraic topology, Cambridge University Press, Cambridge, 2002. MR 1867354

[Ive92] Birger Iversen, Hyperbolic geometry, London Mathematical Society Student Texts, vol. 25, Cambridge University Press, Cambridge, 1992. MR 1205776

[Laf02] Vincent Lafforgue, K-théorie bivariante pour les algèbres de Banach et conjecture de Baum-Connes, Invent. Math. 149 (2002), no. 1, 1-95. MR 1914617

[Lin11] Huaxin Lin, Asymptotic unitary equivalence and classification of simple amenable $C^{*}$ algebras, Invent. Math. 183 (2011), no. 2, 385-450. MR 2772085

[Lor88] Terry A. Loring, K-theory and asymptotically commuting matrices, Canad. J. Math. 40 (1988), no. 1, 197-216. MR 928219

[PS90] Anthony V. Phillips and David A. Stone, The computation of characteristic classes of lattice gauge fields, Comm. Math. Phys. 131 (1990), no. 2, 255-282. MR 1065672

[Rie81] Marc A. Rieffel, $C^{*}$-algebras associated with irrational rotations, Pacific J. Math. 93 (1981), no. 2, 415-429. MR 623572 (83b:46087)

[Sch05] Thomas Schick, $L^{2}$-index theorems, $K K$-theory, and connections, New York J. Math. 11 (2005), 387-443 (electronic). MR 2188248

E-mail address: jcarrion@math.purdue.edu

E-mail address: mdd@math.purdue.edu

Department of Mathematics, Purdue University, West Lafayette, IN, 47907, United STATES 